\title{
HYPERFINITE TRANSVERSAL THEORY
}

\author{
BOŠKO ŽIVALJEVIĆ
}

\begin{abstract}
A measure theoretic version of a well-known P. Hall's theorem, about the existence of a system of distinct representatives of a finite family of finite sets, has been proved for the case of the Loeb space of an internal, uniformly distributed, hyperfinite counting space. We first prove Hall's theorem for $\Pi_{1}^{0}(\kappa)$ graphs after which we develop the version of discrete Transversal Theory. We then prove a new version of Hall's theorem in the case of $\Sigma_{1}^{0}(\kappa)$ monotone graphs and give an example of a $\Sigma_{1}^{0}$ graph which satisfies Hall's condition and which does not possess an internal a.e. matching.
\end{abstract}

\section{INTRODUCTION}

The famous theorem of $\mathrm{P}$. Hall [Ha] gives a necessary and sufficient condition for a bipartite graph $\Gamma$ (a subset of the cartesian product of two finite sets $X$ and $Y$ ) insuring the existence of a 1-1 function $f$, called a matching of $\Gamma$, which is a subset of $\Gamma$ and which has the same domain as $\Gamma$. The theorem is usually called Marriage Theorem. The members of the set $X$ are called "bcys", the members of the set $Y$ "girls", and the bipartite graph $\Gamma$ is interpreted as the preference relation assigning to every "boy" $x$ its set $\Gamma(x)$ of preferred "girls". Hall's condition then, expresses the possibility of marrying every "boy" to a "girl" respecting the preference relation $\Gamma$ in, of course, a one to one manner. The graph $\Gamma$ can also be represented as a finite family of finite sets, simply by replacing $\Gamma$ with the family $\{\Gamma(x): x \in X)\}$. Hall's condition is then, a statement about the existence of a system of distinct representatives of the given family of sets, i.e., an injective choice function. A part of Graph Theory which deals with refinements and variants of P. Hall's theorem is called Transversal Theory (see [Mi]).

P. Hall's theorem has been generalized so far, in two ways. The first direction asks for a necessary and sufficient condition for the existence of a matching for a family of sets whose members are not necessarily finite (see [HPS] for an extensive treatment of the subject).

The second direction however, either replaces the cardinality function in Hall's condition by an abstract "measure" function, as was done in an early paper of R. Rado [Ra] and recently by Bollobas and Varopoulos [BoVa], or, exhibits the "effectiveness" of a matching whose existence is claimed by Hall's

Received by the editors February 8, 1989 and, in revised form, January 13, 1990. Presented at the 95th annual meeting of the Society, Phoenix, Arizona, January 1989.

1980 Mathematics Subject Classification (1985 Revision). Primary 03H05; Secondary 05C70, 28A12, 28A99. 
theorem (see [MaRo] and [Ki]), that is, discusses the "complexity" of the injective function in Hall's theorem.

The approach to Hall's theorem that we adopt here is, in a sense, a combination of these two. The sets $X$ and $Y$ are considered to be Loeb measurable sets (see [StBa] or [HuLo]) with respect to some bounded uniformly distributed hyperfinite counting measure. Hall's condition is replaced by its measure theoretic analog and for the graph $\Gamma$ we choose sets of different "complexity" (in the sense of Descriptive Set Theory) which touches the subjects of Measure Theory and Hyperfinite Descriptive Set Theory (see [KKLM] and [He]). At the same time the choice function in the statement of Hall's theorem is asked to be of "nice" structure, that is we ask that the function is internal. The terminology is likewise adjusted to the measure case situation. Accordingly, a matching need not have the domain equal to the domain of the graph, as it was asked in conclusion of the discrete case of Hall's theorem, but, rather, a domain of full measure in the domain of the graph. These matchings are called almost everywhere (a.e.) matchings.

As we have already said, the idea of replacing, in Hall's theorem, the cardinality of a set by an abstract measure function goes back to R. Rado [Ra] in 1938. He proved a variant of Hall's theorem which deals with selecting subsets of a given finite family of sets combinatorially similar to the given finite family of sets (for the treatment of infinite family of sets in a Loeb space see [ $\left.\mathrm{Zi}_{1}\right]$ ). The problem has been reopened by $\mathrm{C}$. Nash-Williams in his survey paper [NW] and by B. Bollobas [Bo]. Nash-Williams asked, simply, if Hall's theorem holds true for an arbitrary nonatomic measure space or, more correctly, for what measure spaces Hall's theorem is still valid. In that sense, the results that we present here may be regarded as a partial solution to the problem posed by Nash-Williams and B. Bollobas.

After giving necessary terminology, definitions, and notations in $\S 0$ we continue by proving Hall's theorem for a special class of graphs that we call $\Pi_{1}^{0}(\kappa)$ graphs (intersection of $\kappa$ many internal graphs) in $\S 1$. This is a slight extension of a result obtained by D. Ross [Ro].

The new version of Hall's theorem has been used to prove various results for $\Pi_{1}^{0}(\kappa)$ graphs which are, as in the discrete case, the consequences of Hall's theorem. In $\S \S 2-9$ we check that the procedure, usually called the technique of elementary constructions, can be indeed applied in the new situation. The question of what condition is equivalent to the existence of an internal a.e. matching of prescribed measure defect is discussed in $\S 2$ (the defect form of Hall's condition) and the question of when a $\Pi_{1}^{0}(\kappa)$ graph possesses an internal function $\mathscr{M}$, which is a finite union of internal partial matchings and such that the domain of $\mathscr{M}$ is of prescribed measure, is exhibited in $\S 3$ (the partitiondefect form of Hall's theorem). In $\S \S 4-9$ the problem of the existence of an internal a.e. matching of a $\Pi_{1}^{0}(\kappa)$ graph saturating given Loeb measurable sets in the domain or in the range of the given graph is examined. The existence of a matching containing a given Loeb measurable set in $Y$ is discussed in $\S 4$; the existence of full matching containing a given Loeb measurable set in $Y$ in $\S 5$; the existence of a matching containing a given Loeb measurable set in $Y$ and having a prescribed measure defect in $\S 6$; the existence of an internal link of two Loeb measurable sets in $X$ and $Y$ with or without prescribed measure 
defect in $\S \S 8$ and 9 respectively; and finally, the existence of partial matchings of prescribed measure defect and with mutually disjoint ranges is discussed in $\S 9$.

In $\S 10$ the case of $\Sigma_{1}^{0}(\kappa)$ monotone graphs is exhibited. These are the graphs that can be obtained as an increasing union of internal graphs of length $\kappa$. It was shown that if Hall's condition holds then the graph in question possesses internal matchings of arbitrary small measure defect, i.e., the measure of the domain of the matching differs from the measure of the domain of the graph by an in advanced given number. This new variant of Hall's theorem gives rise to an approximation version of Hyperfinite Transversal Theory which was briefly discussed at the end of $\S 10$. It was also shown that this form of Hall's theorem is best possible. In other words, an example of a $\Sigma_{1}^{0}$ graph which satisfies Hall's condition and which however, cannot possess any matching which preserves measure is provided.

The results of this paper are almost entirely based on my doctoral dissertation at the University of Illinois, Urbana-Champaign. I would like to thank my advisor Professor Ward Henson for the generous help he offered during the dissertation research as well as during the preparation of this paper.

\section{BASIC NOTATIONS AND DEFINITIONS}

In this section we give basic notations and definitions from the Theory of Loeb Spaces (see also [StBa]). For an introduction to Nonstandard Analysis the reader is referred to the book of A. Hurd and P. Loeb [HuLo] or to the book of K. Stroyan and W. A. J. Luxemburg [St, Lu].

Let

$$
\mathfrak{A}=(\boldsymbol{\Omega}, \boldsymbol{\Sigma}, \mu)
$$

be an unbounded hyperfinite uniformly distributed counting space. This means that, $\Omega$ is a hyperfinite set, $\Sigma$ is the internal algebra of all internal subsets of $\Omega$, and $\mu$ an internal counting measure on $\Sigma$ defined as

$$
\mu(A)=\frac{|A|}{H},
$$

where $A$ is an internal subset of $\Omega,|A|$ its internal cardinality, and $H$ is an arbitrary but fixed infinite integer.

We refer to the set $\Omega$ as the ground set and suppose that its $\mu$ measure $\mu(\Omega)$ is infinite, which justifies the word "unbounded" in our definition of $\mathfrak{A}$.

The associated Loeb space $L(\mathfrak{A})$ to the internal measure space $\mathfrak{A}$ is a standard measure space

$$
L(\mathfrak{A})=(\Omega, L(\Sigma), L(\mu)),
$$

where $L(\Sigma)$ is, as usual, the $\sigma$-algebra of all Loeb measurable subsets of $\Omega$ and $L(\mu)$ is the $\sigma$-additive, complete measure extending $s t \circ \mu$, st being the standard part map. P. A. Loeb remarked in [Lo] that, if the nonstandard universe is $\aleph_{1}$ saturated, the measure $s t \circ \mu$ satisfies the Caratheodory condition for the extension of countably additive measures and thus can be extended to the measure described above.

The standard product of the measure space $L(\mathfrak{A})$ with itself will be denoted as

$$
L(\mathfrak{A}) \times L(\mathfrak{A})=(\Omega \times \Omega, L(\Sigma) \times L(\Sigma), L(\mu) \times L(\mu)) .
$$


The internal product $\mathfrak{A} \times \mathfrak{A}$ is an internal counting space and its corresponding Loeb space is

$$
L(\mathfrak{A} \times \mathfrak{A})=(\Omega \times \Omega, L(\Sigma \times \Sigma), L(\mu \times \mu)) .
$$

In a similar way we denote the Loeb space product of two not necessarily identical internal counting measure spaces.

As is well known (see for example [StBa]), the space $L(\mathfrak{A} \times \mathfrak{A})$ contains $L(\mathfrak{A}) \times L(\mathfrak{A})$, i.e. $L(\Sigma) \times L(\Sigma) \subseteq L(\Sigma \times \Sigma)$, and the measures $L(\mu) \times L(\mu)$ and $L(\mu \times \mu)$ coincide on $L(\Sigma) \times L(\Sigma)$. The example given by Hoover [StBa] shows that the above inclusion is strict. The sets which are $L(\mu \times \mu)$ or $L(\mu)$ measurable we call simply Loeb measurable sets.

Given an infinite cardinal $\kappa$, a $\Pi_{1}^{0}(\kappa)$ set is an intersection of $\kappa$ many internal sets and a $\Sigma_{1}^{0}(\kappa)$ monotone set is an increasing union of $\kappa$ many internal sets. When the cardinal $\kappa$ is equal to the first infinity cardinal $\omega$ then a $\Sigma_{1}^{0}(\kappa)$ monotone set is just a union of countably many internal sets. Let $G$ be arbitrary internal set. A Borel set in $G$ is just an element of the least $\sigma$ algebra containing the algebra of all internal subsets of $G$. Let $\omega^{<\omega}$ be the set of all finite sequences of natural numbers and let $\omega^{\omega}$ be the set of all infinite sequences of natural numbers. A Souslin set in $G$ is a set that can be obtained as

$$
\bigcup_{\xi \in \omega^{\omega}} \bigcap_{n \in \omega} W_{\xi \backslash n},
$$

where $W_{s} \quad\left(s \in \omega^{<\omega}\right)$ is a family of internal subsets of $G$. A Souslin (Borel) function is a function whose graph is Souslin (Borel) in the product of two internal sets.

Given Loeb measurable sets $X$ and $Y$, a bipartite graph or, more simply, a graph is just an arbitrary subset of the cartesian product $X \times Y$. The elements of the set $X \cup Y$ are called vertices or nodes and the elements of $\Gamma$ are called edges.

A graph $\Gamma$ can be pictured simply as a family of sets. We represent each node, $x \in X$, as tile set $\Gamma_{x}=\{y \in Y:(x, y) \in \Gamma\}$ (the $Y$-section of $x$ with respect to $\Gamma$ ), and the whole graph $\Gamma$ is now a family $\left\{\Gamma_{x}: x \in X\right\}$. This point of looking at graphs is useful in what follows. Also, if the graph $\Gamma$ is Borel, Souslin, Loeb measurable or of some other type, then we call the corresponding family by the same name (Borel, Souslin, Loeb measurable, etc.).

If $A \subseteq X$ is an arbitrary subset of $X$ then the image $\Gamma(A)$ of the set $A$ under $\Gamma$ is defined as

$$
\Gamma(A)=\{y:(\exists x \in A)(x, y) \in \Gamma\} .
$$

The inverse graph $\Gamma^{-1} \subseteq Y \times X$ is defined as

$$
(y, x) \in \Gamma^{-1} \text { if and only if }(x, y) \in \Gamma \text {. }
$$

The domain, $\operatorname{dom}(\Gamma)$, and the range $\operatorname{ran}(\Gamma)$, of $\Gamma$ are defined as $\operatorname{dom}(\Gamma)=$ $\Gamma(X), \operatorname{ran}(\Gamma)=\Gamma^{-1}(Y)$.

A (partial) matching $\mathscr{M}$ of $\Gamma$ is an injective function whose graph is a subset of $\Gamma$. We say that a vertex $v$ is saturated by $\mathscr{M}$ if $v$ is either in the domain or range of $\Gamma$. Otherwise a vertex is called unsaturated. 
The main question, throughout this paper, is the following:

Given a certain kind of graph

$$
\Gamma \subseteq X \times Y,
$$

$\left(L(\mu \times \mu)\right.$ measurable, Souslin, $\Pi_{1}^{0}(\kappa)$, or some other) what necessary and (or) sufficient condition must one impose on $\Gamma$ such that $\Gamma$ possesses a certain kind of matching (internal, $\Sigma_{1}^{0}$, Borel, or some other).

Let us remark here that we may concentrate on internal a.e. matchings only, for by a result of Henson and Ross (see [HeRo] and [ $\left[\mathrm{Z}_{2}\right]$ ) every Souslin (and injective) function is $L(\mu)$ a.e. equal to an internal (and injective) function.

In the case of standard Transversal Theory, which is the case when our infinite integer $H$ actually equals 1 , and the measure $\mu$, and thus $L(\mu)$, is equal to the cardinality of a set, the answer is provided by the following famous and well-known theorem due to P. Hall. For the proof see [Mi] or [Ha].

Theorem (P. Hall). Let $X$ and $Y$ be finite sets and let $\Gamma \subseteq X \times Y$ be a bipartite graph. Then, there exists a matching $\mathscr{M} \subseteq \Gamma$ saturating all vertices in $X$ if and only if $|\Gamma(A)| \geq|A|$ for every subset $A$ of $X$.

Actually, in what follows, we shall need the following, so called defect form of Hall's theorem, which is an easy consequence of Hall's theorem and is usually credited to P. Hall and O. Ore. Again, for the proof we refer to the book of Mirsky [Mi].

Theorem (P. Hall-O. Ore). Let $X$ and $Y$ be finite sets and let $\Gamma \subseteq X \times Y$ be a bipartite graph. Then, there exists a matching $\mathscr{M} \subseteq \Gamma$ such that the number of vertices in $X$ unsaturated by $\mathscr{M}$ is less than or equal to $d$ if and only if $|\Gamma(A)| \geq|A|-d$ for every subset $A$ of $X$.

The conditions in P. Hall's and P. Hall and O. Ore's theorem are usually called Hall's and defect Hall's condition respectively. We adopt the same name for the measure analog of the same condition. More precisely, given Loeb measurable sets $X$ and $Y$ and a graph $\Gamma$,

$$
\Gamma \subseteq X \times Y,
$$

we say that $\Gamma$ satisfies Hall's condition if

$$
L_{i}(\mu)(\Gamma(A)) \geq L(\mu)(A),
$$

for every internal $A \subseteq X$, or

$$
L_{e}(\mu)(\Gamma(A)) \geq L(\mu)(A),
$$

for every internal $A \subseteq X$. The choice of the inner $L_{i}(\mu)$ or outer $L_{e}(\mu)$ measure will vary from case to case. Most often, $\Gamma$ maps internal sets into measurable ones, and the meaning of Hall's condition is clear, and similarly for the defect Hall's condition.

Throughout this paper the term Loeb measurable will always refer to $L(\mu)$ measurable if otherwise is not stated. Also, we assume that our nonstandard universe is $\kappa^{+}$saturated. 


\section{HALL'S THEOREM FOR $\Pi_{1}^{0}(\kappa)$ GRAPHS}

In this section we prove that Hall's condition for a $\Pi_{1}^{0}(\kappa)$ graph is equivalent to the existence of an internal matching saturating almost all of the domain of a graph. Then, we given another formulation of Hall's condition in terms of the inverse images of internal sets.

Before we state and prove the results, we need the following technical fact which was noticed before by many authors (see [LaRo] for example).

Lemma 1.1. Let $A_{\alpha}(\alpha<\kappa)$ be a sequence of internal subsets of $\Omega$ contained in an internal set of finite $L(\mu)$ measure. Then, $\bigcup_{\alpha<\kappa} A_{\alpha}$ and $\bigcap_{\alpha<\kappa} A_{\alpha}$ are Loeb measurable. If these families are directed above (below), then

$$
\begin{aligned}
& L(\mu)\left(\bigcup_{\alpha<\kappa} A_{\alpha}\right)=\sup \left\{L(\mu)\left(A_{\alpha}\right): \alpha<\kappa\right\}, \\
& L(\mu)\left(\bigcap_{\alpha<\kappa} A_{\alpha}\right)=\inf \left\{L(\mu)\left(A_{\alpha}\right): \alpha<\kappa\right\} .
\end{aligned}
$$

Proof. It is sufficient to prove that $\bigcup_{\alpha<\kappa} A_{\alpha}$ is Loeb measurable. We may assume that the family $A_{\alpha}(\alpha<\kappa)$ has f.i.p. for otherwise the intersection $\bigcap_{\alpha<\kappa} A_{\alpha}$ would be empty and the result would be trivial. Also, we may suppose that the family $A_{\alpha}(\alpha<\kappa)$ is closed with respect to finite intersections. Let $a$ be the outer measure of $\bigcap_{\alpha<\kappa} A_{\alpha}$. If $B \supseteq \bigcap_{\alpha<\kappa} A_{\alpha}$ is an internal set then, by saturation, $B \supseteq A_{\beta}$ for some $\beta$, and, consequently, $a=\inf \left\{L(\mu)\left(A_{\alpha}\right): \alpha<\right.$ $\kappa\}$. Given $\varepsilon>0$ and $\alpha<\kappa$ let

$$
\mathscr{S}_{\alpha}=\left\{B \in \Sigma: B \subseteq A_{\alpha}, \mu(B) \geq a-\varepsilon\right\} .
$$

It is clear that this family possesses f.i.p., which shows that the inner measure of $\bigcap_{\alpha<\kappa} A_{\alpha}$ is $\geq a-\varepsilon$ and which in turn proves our claim.

We also need the following two lemmas. The first of them (Lemma 1.2) was observed by Henson in [He] and the second one (Lemma 1.3) is the immediate consequence of the first.

Lemma 1.2. Let $\Gamma \subseteq \Omega \times \Omega$, be a graph such that the inverse image $\Gamma^{-1}(x)$ of each point $x$ is a $\Pi_{1}^{0}(\kappa)$ set. Let $A_{\alpha}(\alpha<\kappa)$ be a sequence of internal sets closed with respect to finite intersections. Then

$$
\Gamma\left(\bigcap_{\alpha<\kappa} A_{\alpha}\right)=\bigcap_{\alpha<\kappa} \Gamma\left(A_{\alpha}\right) .
$$

Proof. The inclusion

$$
\Gamma\left(\bigcap_{\alpha<\kappa} A_{\alpha}\right) \subseteq \bigcap_{\alpha<\kappa} \Gamma\left(A_{\alpha}\right)
$$

is obvious.

To prove the converse let $a \in \bigcap_{\alpha<\kappa} \Gamma\left(A_{\alpha}\right)$. Then, from the fact that $\Gamma^{-1}(a)$ is $\Pi_{1}^{0}(\kappa)$, we have 


$$
\Gamma^{-1}(a)=\bigcap_{\alpha<\kappa} B_{\alpha}
$$

for some family $B_{\alpha}(\alpha<\kappa)$ of internal sets for which we may assume that is closed with respect to finite intersections. Now, the condition

$$
\Gamma^{-1}(a) \cap A_{\alpha} \neq \varnothing,
$$

for every $\alpha<\kappa$, insures that the family $\left\{A_{\alpha} \cap B_{\beta}: \alpha, \beta<\kappa\right\}$, possesses f.i.p. and thus, has nonempty intersection. An element $b \in \bigcap_{\alpha, \beta}\left(A_{\alpha} \cap B_{\beta}\right)$ has the property $\Gamma(b)=a$, so

$$
a \in \Gamma\left(\bigcap_{\alpha<\kappa} A_{\alpha}\right) \text {. }
$$

The typical application of the previous lemma is when $\Gamma$ is an internal or $\Pi_{1}^{0}(\kappa)$ graph and $f$ is the projection map. The condition that the inverse images of points are $\Pi_{1}^{0}(\kappa)$ sets is obviously satisfied in that case.

Lemma 1.3. Let

$$
\Gamma \subseteq \Omega \times \Omega, \quad \Gamma=\bigcap_{\alpha<\kappa} \Gamma_{\alpha}
$$

be a $\Pi_{1}^{0}(\kappa)$ graph and let $P \subseteq \Omega, P=\bigcap_{\alpha<\kappa} A_{\alpha}$ be a $\Pi_{1}^{0}(\kappa)$ set. Suppose that $\Gamma_{\alpha}$ and $A_{\alpha}$ are closed with respect to finite intersections. Then

$$
\Gamma\left(\bigcap_{\alpha<\kappa} A_{\alpha}\right)=\bigcap_{\alpha, \beta} \Gamma_{\beta}\left(A_{\alpha}\right)
$$

In particular, the domain and the range of a $\Pi_{1}^{0}(\kappa)$ graph are $\Pi_{1}^{0}(\kappa)$ sets.

Proof. By Lemma 1.2 we have

$$
\Gamma\left(\bigcap_{\alpha<\kappa} A_{\alpha}\right)=\bigcap_{\alpha<\kappa} \Gamma\left(A_{\alpha}\right)
$$

Moreover, if $\pi_{2}: \Omega \times \Omega \rightarrow \Omega$ is the projection map to the second coordinate, then

$$
\Gamma\left(A_{\alpha}\right)=\pi_{2}\left(\left(A_{\alpha} \times \Omega\right) \cap \Gamma\right)=\pi_{2}\left(\left(A_{\alpha} \times \Omega\right) \cap\left(\bigcap_{\beta<\kappa} \Gamma_{\beta}\right)\right) .
$$

Note that when $\alpha$ is fixed, $\left(A_{\alpha} \times \Omega\right) \cap \Gamma_{\beta}$ is closed with respect to finite intersections. Applying Lemma 1.2 once again, we obtain the result.

Hall's theorem for internal graphs was first proved by D. Ross [Ro]. We repeat the proof here, then extend it by allowing $\Gamma$ to be a $\Pi_{1}^{0}(\kappa)$ graph and then give another necessary and sufficient condition for the existence of an internal matching saturating almost all of the domain of a graph. Lemmas 1.1, 1.2, and 1.3 insure that if $A$ is an internal set and $\Gamma$ a $\Pi_{1}^{0}(\kappa)$ graph, then $\Gamma(A)$ is always Loeb measurable. Recall that a $\Pi_{1}^{0}(\kappa)$ graph is just an intersection of $\kappa$ many internal graphs. 
Proposition 1.4. Let $X$ and $Y$ be Loeb measurable sets of finite measure and let $\Gamma \subseteq X \times Y$ be a $\Pi_{1}^{0}(\kappa)$ graph. Then, $\Gamma$ has an internal matching saturating almost all elements of $X$ if and only if Hall's condition holds for $\Gamma$, i.e.

$$
L(\mu)(\Gamma(A)) \geq L(\mu)(A),
$$

for every internal subset $A$ of $X$.

Proof. The necessity of the above condition is obviously true. Let us prove its sufficiency.

We shall first suppose that $\Gamma, X$ and $Y$ are internal. Let

$$
\beta=\sup \left\{\frac{|A|-|\Gamma(A)|}{H}: A \subseteq X \text { internal }\right\},
$$

be the internal supremum of the set from the right. Then, condition $(\mathscr{H})$ insures that either $\beta$ is negative or is positive and infinitesimal. In the first case we have $|\Gamma(A)| \geq|A|$ for every internal subset $A \subseteq X$, and the result follows from Hall's theorem and the Transfer Principle. If $\beta$ is positive and infinitesimal then,

$$
|\Gamma(A)| \geq|a|-\beta \cdot H,
$$

for every internal $A \subseteq X$ and, again, by Hall-Ore's theorem and the Transfer Principle, $\Gamma$ possesses an internal matching such that the number of unsaturated vertices is $\leq[\beta \cdot H]$, where $[\beta \cdot H]$ is the least integer greater or equal to $\beta \cdot H$. But, $[\beta \cdot H]-1 \leq \beta \cdot H$ and thus, $[\beta \cdot H] / H$ is infinitesimal, which proves our claim.

Suppose now, that the graph $\Gamma$ is $\Pi_{1}^{0}(\kappa)$, i.e. $\Gamma$ is of the form

$$
\Gamma=\bigcap_{\alpha<\kappa} \Gamma_{\alpha},
$$

where $\Gamma_{\alpha}(\alpha<\kappa)$ are internal graphs, and that $X$ and $Y$ are Loeb measurable ( $X$ being of finite measure). Let $A_{n} \subseteq X$ be an increasing sequence of internal subsets of $X$ such that

$$
L(\mu)(X)-L(\mu)\left(A_{n}\right) \leq 1 / n .
$$

We may assume that the sequence $\Gamma_{\alpha}(\alpha<\kappa)$ is closed with respect to finite intersections. Following the fact that $\Gamma$ satisfies Hall's condition $(\mathscr{H})$ and that $\Gamma \subseteq \Gamma_{\alpha}(\alpha<\kappa)$, each of the graphs $\Gamma_{\alpha} \mid A_{n}=\Gamma_{\alpha} \cap\left(A_{n} \times \Omega\right)$ satisfies Hall's condition. Therefore, by the first part of the proof, each of the graphs $\Gamma_{\alpha}\left\lceil A_{n}\right.$ has an internal matching with domain of full measure in $A_{n}$. That insures that the family

$$
\begin{aligned}
& \mathscr{S}_{\alpha, m, n}=\left\{\mathscr{M}: \mathscr{M} \subseteq \Gamma_{\alpha} \mid A_{n} \text { an internal } 1-1\right. \text { function, } \\
&\left.\mu\left(A_{n}\right)-\mu(\operatorname{dom} \mathscr{M}) \leq 1 / m\right\},
\end{aligned}
$$

possesses f.i.p. and thus, has nonempty intersection. An element of that intersection is a matching of $\Gamma$ which saturates almost all elements of $\bigcup_{n} A_{n}$ and thus, almost all elements of $X$.

The following proposition is another formulation of Hall's condition and it has its counterpart in the standard Transversal Theory one can find in [Be]. 
Proposition 1.5. Let $X$ and $Y$ be Loeb measurable sets of finite measure and let

$$
\Gamma \subseteq X \times Y
$$

be a $\Pi_{1}^{0}(\kappa)$ graph. Then, $\Gamma$ has an internal matching saturating almost all $X$ if and only if

$$
L(\mu)\left(X \backslash \Gamma^{-1}(B)\right) \leq L(\mu)(Y \backslash B)
$$

for every internal $B \subseteq Y$.

Proof. $\Rightarrow$ : Let us suppose that $\Gamma$ has an internal matching saturating almost all $X$. We shall prove that the above condition is satisfied.

Assume first, that $X$ is internal. Let $B \subseteq Y$ be an internal set. Now, note that Hall's condition is satisfied for the graph $\Gamma$ and, by Lemma 2.1 (§2), that it holds for $\Sigma_{1}^{0}(\kappa)$ set $X \backslash \Gamma^{-1}(B)$. Therefore, we have

$$
L(\mu)\left(X \backslash \Gamma^{-1}(B)\right) \leq L_{i}(\mu)\left(\Gamma\left(X \backslash \Gamma^{-1}(B)\right) \leq L(\mu)(Y / B),\right.
$$

where the last inequality holds because $\Gamma\left(X \backslash \Gamma^{-1}(B)\right) \subseteq Y \backslash B$.

For a general Loeb measurable $X$ we choose an internal set $D$ with $L(\mu)(D \Delta X)=0$, and apply the first part of the proof to $\Gamma \cap(D \times \Omega)$.

$\Leftarrow$ : To prove that $\Gamma$ has an internal matching saturating almost all $X$ we need to prove that Hall's condition holds. Suppose not. Then, there exists an internal set $A_{0} \subseteq X$ with

$$
L(\mu)\left(\Gamma\left(A_{0}\right)\right)<L(\mu)\left(A_{0}\right) .
$$

Let $M=Y \backslash \Gamma\left(A_{0}\right)$. We have $A_{0} \subseteq X \backslash \Gamma^{-1}(M)$ so,

$$
L(\mu)\left(X \backslash \Gamma^{-1}(M)\right) \geq L(\mu)\left(A_{0}\right)>L(\mu)(\Gamma(A))=L(\mu)(Y \backslash M),
$$

which is a contradiction if the condition similar to $(*)$ holds not only for every internal set $B$ but for every Loeb measurable set $M$. Indeed, we prove that if $(*)$ holds for every internal $B$ then

$$
L_{e}(\mu)\left(X \backslash \Gamma^{-1}(M)\right) \leq L(\mu)(Y \backslash M),
$$

for every Loeb measurable $M \subseteq Y$.

Take any internal $B \subseteq M$ then, $X \backslash \Gamma^{-1}(B) \supseteq X \backslash \Gamma^{-1}(M)$. Therefore,

$$
L_{e}(\mu)\left(X \backslash \Gamma^{-1}(M)\right) \leq L(\mu)\left(X \backslash \Gamma^{-1}(B)\right) \leq L(\mu)(Y \backslash B) .
$$

If we take the supremum over all internal $B \subseteq M$, we obtain the result.

\section{THE DEFECT FORM OF HALl'S THEOREM FOR $\Pi_{1}^{0}(\kappa)$ GRAPHS}

We are ready now to use our Proposition 1.4 and to prove a number of results that are, as in standard Transversal Theory, consequences of Hall's theorem. We show that a similar technique to one used in the standard setting, and which is usually called the method of elementary constructions, leads to analogous answers. For the standard treatment we refer to a book of Mirsky [Mi].

We begin with the defect form of Hall's theorem which in the discrete case is, as we mentioned before, due to P. Hall and O. Ore. First, we need a technical fact. 
Lemma 2.1. Let $\Gamma$ be a graph in $X \times Y$,

$$
\Gamma \subseteq X \times Y,
$$

where $X$ and $Y$ are Loeb measurable sets of finite measure. Suppose that the defect form of Hall's condition is satisfied, i.e.

$$
L(\mu)_{i}(\Gamma(A)) \geq L(\mu)(A)-r,
$$

for every internal subset $A$ of $X$ and a fixed real number $r$. Then,

$$
L(\mu)_{i}(\Gamma(M)) \geq L(\mu)_{i}(M)-r,
$$

for every subset $M$ of $X, M \subseteq X$, where $L(\mu)_{i}(\Gamma(M))$ is, as usual, the inner measure of $\Gamma(M)$ and $L(\mu)_{i}(M)$ is the inner measure of $M$.

Proof. Indeed, let $M$ be a Loeb measurable subset of $X$ and $A \subseteq M$ an internal set. Then, by Hall's condition, we have

$$
L(\mu)_{i}(\Gamma(M)) \geq L(\mu)_{i}(\Gamma(A)) \geq L(\mu)(A)-r,
$$

and the result follows by taking the supremum over all internal subsets $A$ of $M$.

Proposition 2.2. Let $X$ and $Y$ be Loeb measurable sets of finite measure and let $r$ be a real number. Let

$$
\Gamma \subseteq X \times Y,
$$

be a $\Pi_{1}^{0}(\kappa)$ graph. Then, $\Gamma$ has an internal matching such that the measure of the set of unsaturated vertices in $X$ is less than or equal to $r$ if and only if

$$
L(\mu)(\Gamma(A)) \geq L(\mu)(A)-r,
$$

for every internal subset $A$ of $X$.

Proof. Suppose that the above condition is satisfied. Let $D \subseteq \Omega$ be an internal set of Loeb measure $r$, and such that $D \cap Y=\varnothing$. Let $P$ be a $\Pi_{1}^{0}$ set containing $X$ and of the same measure as $X$, i.e. $P \supseteq X, L(\mu)(P)=L(\mu)(X)$. The graph

$$
\Gamma^{\prime}=\Gamma \cup P \times D,
$$

is a $\Pi_{1}^{0}(\kappa)$ graph which satisfies Hall's condition in $P$. Indeed, let $A \subseteq P$ be an internal set. Then, by the fact that $\Gamma$ satisfies the above condition and by Lemma 2.1, we have

$$
L(\mu)(A)=L(\mu)(A \cap X) \leq L(\mu)(\Gamma(A \cap X))+r=L(\mu)\left(\Gamma^{\prime}(A)\right),
$$

for every internal and nonempty subset $A$ of $P$. Thus, by Proposition $1.2 \Gamma^{\prime}$ has an internal matching $\mathscr{G}$ which saturates almost all elements of $P$ and the function $\mathscr{K}=\mathscr{G} \backslash(\Omega \times D)$ is the desired matching.

Conversely, if $\Gamma$ possesses an internal matching such that the measure of unsaturated vertices in $X$ is less or equal to $r$, then, obviously, the condition $(\mathscr{D} \mathscr{H})$ is satisfied.

The condition $(\mathscr{D} \mathscr{H})$ is usually called the defect form of Hall's condition. 
Definition 2.3. Let $X$ and $Y$ be Loeb measurable sets and let $\Gamma \subseteq X \times Y$ be an arbitrary graph. The measure defect $\delta(\Gamma)$ of $\Gamma$ is defined to be the number:

$$
\begin{array}{r}
\delta(\Gamma)=\inf \{L(\mu)(A): A \subseteq X \text { internal, } \Gamma \text { has an internal matching } \\
\text { saturating almost all of } X \backslash A\} .
\end{array}
$$

Note that the definition of the measure defect of $\Gamma$ depends on the set $X$. The following characterization of the measure defect of $\Pi_{1}^{0}(\kappa)$ graphs is an immediate consequence of the defect form of Hall's theorem (Proposition 2.2).

Proposition 2.4. Let $X$ and $Y$ be Loeb measurable sets of finite measure. Let $\Gamma \subseteq X \times Y$ be a $\Pi_{1}^{0}(\kappa)$ graph. Then,

$$
\delta(\Gamma)=\sup \{L(\mu)(A)-L(\mu)(\Gamma(A)): A \subseteq X \text { internal }\} .
$$

\section{THE PARTITION-DEFECT FORM OF HALL'S THEOREM FOR $\Pi_{1}^{0}(\kappa)$ GRAPHS}

Hall's condition gives a necessary and sufficient condition for a $\Pi_{1}^{0}(\kappa)$ graph $\Gamma$ to possess an internal matching saturating the set $X$ almost everywhere. The next proposition deals with the question of when can we partition the ground set $X$ into finitely many sets in such a way that our graph $\Gamma$ restricted to any of these sets has an internal partial matching such that the global measure defect of these matchings is within a prescribed bound. The answer to this question in the discrete case was provided by R. Rado and it can be found in the already mentioned book of Mirsky.

Proposition 3.1. Let $X$ and $Y$ be Loeb measurable sets of finite measure. Let $\Gamma \subseteq X \times Y$ be a $\Pi_{1}^{0}(\kappa)$ graph and $r$ a real number. Then, the following conditions are equivalent:

(i) $X$ can be partitioned into $n$ Loeb measurable sets $X=\bigcup_{i=1}^{n} X_{i}$ such that there exists an internal function $\mathscr{M}$ in $\Gamma$ which restriction to each of the members of the partition is an injective function and such that the measure of the set of vertices in $X$ nonsaturated by $\mathscr{M}$ is less than or equal to $r$;

(ii)

$$
n \cdot L(\mu)(\Gamma(A)) \geq L(\mu)(A)-r,
$$

for every internal subset $A \subseteq X$ of $X$.

Proof. Let $D$ be a finite set of cardinality $n$ and let $\nu$ be a measure in $D$ defined as $\nu(A)=|A|, A \subseteq D$. In such a way we obtain an internal measure space $(D, \mathfrak{P}, \nu)$.

As usual, to the above space we can associate the corresponding Loeb measure space which is, in this case, the same as the old one.

Let us define a new graph $\Gamma^{\prime}$ in $X \times(Y \times D)$ as $\Gamma^{\prime}=\Gamma \times D$.

In the product $\Omega \times D$ we introduce the measure $L(\mu \times \nu)$, and observe that for a $L(\mu)$ measurable set $M$ and a subset $A$ of $D$

$$
L(\mu \times \nu)(M \times A)=L(\mu)(M) \cdot|A|,
$$

and in particular

$$
L(\mu \times \nu)(M \times D)=L(\mu)(M) \cdot n .
$$


It is easily seen that the graph $\Gamma^{\prime}$ is $\Pi_{1}^{0}(\kappa)$ in $X \times(Y \times D)$.

Let the inequality $(\mathscr{P} \mathscr{D})$ be satisfied. Then, the defect form of Hall's condition for the graph $\Gamma^{\prime}$ is satisfied. Indeed,

$$
\begin{aligned}
L(\mu \times \nu)\left(\Gamma^{\prime}(A)\right) & =L(\mu \times \nu)(\Gamma(A) \times D)=L(\mu)(\Gamma(A)) \cdot n \\
& \geq L(\mu)(A)-r .
\end{aligned}
$$

Thus, $\Gamma^{\prime}$ possesses an internal matching of $\mathscr{G}$ of measure defect $\leq r$. Now, for every $k=1, \ldots, n$ we define

$$
\mathscr{M}_{k}=\{(x, y) \in \Gamma:(x, y, k) \in \mathscr{G}\}=\mathscr{G}^{-1}(k) .
$$

From the fact that $\mathscr{G}$ is an internal injection (that is for every $x \in X$ there exists at most one pair $(y, k)$ such that $(x, y, k) \in \mathscr{G})$, the functions $\mathscr{M}_{k}$ are also internal injections whose domains are mutually disjoint. Thus, if we add the nonsaturated set of measure $\leq r$ to the domain of an arbitrary and fixed function $\mathscr{M}_{k}$ we get the desired partition of $X$, where the function in the claim of our proposition is

$$
\mathscr{M}=\bigcup_{k=1}^{n} \mathscr{M}_{k}
$$

Conversely, if $\mathscr{M}=\bigcup_{k=1}^{n} \mathscr{M}_{k}$ is an internal function in $\Gamma$ and $X=\bigcup_{k=1}^{n} X_{k}$ a partition of $X$ into Loeb measurable sets such that the function $\mathscr{M}_{k}$ are internal and injective with the domains in $X_{k}$ and such that the measure of the vertices in $X$ not saturated by $\mathscr{M}$ is less than $r$, then

$$
\mathscr{G}=\bigcup_{k=1}^{n}\left(\mathscr{M}_{k} \times\{k\}\right)
$$

is a matching of $\Gamma^{\prime}$ of measure defect $\leq r$, so the inequality $(\mathscr{P D})$ is satisfied.

We extract a special case of the preceding proposition separately.

Corollary 3.2. Let $X$ and $Y$ be Loeb measurable sets of finite measure. Let $\Gamma \subseteq X \times Y$ be a $\Pi_{1}^{0}(\kappa)$ graph. Then, $X$ can be partitioned into $n$ Loeb measurable parts $X=\bigcup_{i=1}^{n} X_{i}$ such that there exists an internal function $\mathscr{M}$ in $\Gamma$ whose restriction to each of the members of the partition is injective and such that $\mathscr{M}$ saturates almost all $X$, if and only if

$$
n \cdot L(\mu)(\Gamma(A)) \geq L(\mu)(A)
$$

for every internal subset $A \subseteq X$ of $X$.

Corollary 3.3. Let $X$ and $Y$ be Loeb measurable sets of finite measure and let $\Gamma \subseteq X \times Y$ be a $\Pi_{1}^{0}(\kappa)$ graph. Let

$$
\inf \left\{\frac{L(\mu)(\Gamma(A))}{L(\mu)(A)}: A \subseteq X \text { internal, } L(\mu)(A) \neq 0\right\}=r \neq 0 .
$$

Suppose that $n$ is an integer satisfying $n>1 / r$. Then, $X$ can be partitioned into $n$ Loeb measurable parts $X=\bigcup_{i=1}^{n} X_{i}$ such that there exists an internal function $\mathscr{M}$ in $\Gamma$ whose restriction to each of the members of the partition is injective and such that $\mathscr{M}$ saturates almost all of $X$. 
Definition 3.4. Let $X$ and $Y$ be Loeb measurable sets of finite measure and let $\Gamma \subseteq X \times Y$ be a Loeb measurable graph. The degree $\mathfrak{d}(x) \quad(\mathfrak{d}(y))$ of a point $x \in X \quad(y \in Y)$ is

$$
\mathfrak{d}(x)=L(\mu)(\Gamma(x)), \quad\left(\mathfrak{d}(y)=L(\mu)\left(\Gamma^{-1}(y)\right)\right),
$$

if the corresponding section $\Gamma(x)\left(\Gamma^{-1}(y)\right)$ is Loeb measurable.

The $X(Y)$ degree function of $\Gamma$ is the function $x \rightarrow \mathfrak{d}(x) \quad(y \rightarrow \mathfrak{d}(y))$, the restriction of the function $\mathfrak{d}$ to $X(Y)$.

$\Gamma$ is said to be (a.e.) $r$-regular if $\mathfrak{d}(\alpha)=r$ for all (a.e.) $\alpha \in X \cup Y$.

Note that, by the Keisler's Fubini theorem (see [StBa] for example), the degree function of a Loeb measurable graph is always a.e. defined and Loeb measurable. Also, we can think of the degree function of a graph as of a function that measures the width and the height of the graph, or the degree of choice to select the elements of our desired matching. The following proposition says that if we can bound the degree of a $\Pi_{1}^{0}(\kappa)$ graph from above and from below then, depending on our achieved bounds, the graph possesses a certain kind of a matching.

Proposition 3.5. Let $X$ and $Y$ be Loeb measurable sets of finite measure. Let $\Gamma \subseteq X \times Y$ be a $\Pi_{1}^{0}(\kappa)$ graph. Suppose that

$$
\mathfrak{d}(\beta) \leq r_{1} \text { a.e. on } Y \text { and } \mathfrak{d}(\alpha) \geq r_{2} \text { a.e. on } X .
$$

Then, $X$ can be partitioned into $\left[r_{1} / r_{2}\right]$ Loeb measurable parts such that there exists an internal function $\mathscr{M}$ in $\Gamma$ whose restriction to each of the members of the partition is injective and such that $\mathscr{M}$ saturates almost all of $X$ (recall that $\left[r_{1} / r_{2}\right]$ is the least integer greater or equal to $\left.r_{1} / r_{2}\right)$.

Proof. Let $\Theta \subseteq X$ be internal. Let $M_{1}$ and $M_{2}$ be the sets of edges incident to $\Theta$ and $\Gamma(\Theta)$ respectively. In other words,

$$
M_{1}=(\Theta \times Y) \cap \Gamma, \quad M_{2}=(X \times \Gamma(\Theta)) \cap \Gamma .
$$

We obviously have the inclusion $M_{1} \subseteq M_{2}$. Now, note that the graph $\Gamma$ is, as an intersection of $\kappa$ many internal sets, Loeb measurable (Lemma 1.1) and thus, we can apply Keisler-Fubini theorem to $\Gamma$. We have

$$
L(\mu \times \mu)\left(M_{1}\right)=\int_{\boldsymbol{\Theta}} \mathfrak{d}(\cdot) d L(\mu),
$$

and

$$
L(\mu \times \mu)\left(M_{2}\right)=\int_{\Gamma(\boldsymbol{\theta})} \mathfrak{d}(\cdot) d L(\mu) .
$$

Using the given bounds on the degree function and inclusion $M_{1} \subseteq M_{2}$, we get

$$
r_{2} \cdot L(\mu)(\boldsymbol{\Theta}) \leq \int_{\boldsymbol{\Theta}} \mathfrak{d}(\cdot) d L(\mu) \leq \int_{\Gamma(\boldsymbol{\Theta})} \mathfrak{d}(\cdot) d L(\mu) \leq r_{1} \cdot L(\mu)(\Gamma(\boldsymbol{\Theta})) .
$$

So,

$$
\frac{r_{1}}{r_{2}} \cdot L(\mu)(\Gamma(\Theta)) \geq L(\mu)(\Theta) .
$$


That is

$$
\left[\frac{r_{1}}{r_{2}}\right] \cdot L(\mu)(\Gamma(\boldsymbol{\theta})) \geq L(\mu)(\boldsymbol{\theta}),
$$

for every internal $\boldsymbol{\theta} \subseteq X$. The result now follows from Corollary 3.2.

Recall that the essential supremum $\mathfrak{s}(f)$ and the essential infimum $\mathfrak{i}(f)$ of a Loeb measurable function $f: \Omega \rightarrow \mathbb{R}$ are defined as

$$
\mathfrak{s}(f)=\inf \left\{r: L(\mu)\left(f^{-1}(] r,+\infty[)=0\right\}\right.
$$

and

$$
\mathfrak{i}(f)=\sup \left\{r: L(\mu)\left(f^{-1}(]-\infty, r[)=0\right\} .\right.
$$

The numbers $\mathfrak{s}(\mathfrak{d} \mid X)$ and $\mathfrak{i}(\mathfrak{d} \mid Y)$ measure the "width" and the "height" of the graph. The following corollary justifies what is intuitively clear, namely, if the "height" of the graph is greater than its "width" and the graph is $\Pi_{1}^{0}(\kappa)$ then it possesses an internal matching of zero measure defect.

Corollary 3.6. Let $X$ and $Y$ be Loeb measurable sets of finite measure. Let $\Gamma \subseteq X \times Y$ be a $\Pi_{1}^{0}(\kappa)$ graph and $\mathfrak{d} \mid X(\mathfrak{d} \mid Y)$ the restriction of the degree function $\mathfrak{d}$ to $X(Y)$. Suppose that

$$
\mathfrak{s}(\mathfrak{d} \mid Y) \leq \mathfrak{i}(\mathfrak{d} \mid X)
$$

Then, $\Gamma$ has an internal matching saturating almost all elements of $X$.

Proof. Let $\mathfrak{s}(\mathfrak{d} \mid Y)=r_{1}$ and $\mathfrak{i}(\mathfrak{d} \mid X)=r_{2}$. Then

$$
\mathfrak{d}(\beta) \leq r_{1} \text { a.e. on } Y \text { and } \mathfrak{d}(\alpha) \geq r_{2} \text { a.e. on } X,
$$

and $r_{1} \leq r_{2}$. Thus, $\left[r_{1} / r_{2}\right]=1$ and the result follows from Proposition 3.5.

The case when a $\Pi_{1}^{0}(\kappa)$ graph has equal height and width, i.e. when it is regular, is especially simple.

Corollary 3.7. Let $X$ and $Y$ be Loeb measurable sets of finite measure and let $\Gamma \subseteq X \times Y$ be $\Pi_{1}^{0}(\kappa)$ graph. Suppose that $\Gamma$ is $r$-regular, that is

$$
\mathfrak{d}(x)=r \text { a.e. on } X \cup Y .
$$

Then, $\Gamma$ has an internal matching saturating almost all of $X$ and $Y$.

Proof. By Corollary 3.6, $\Gamma$ has an internal matching $\mathscr{M}$ saturating almost all elements of the set $X$. But,

$$
r \cdot L(\mu)(X)=\int_{X} \mathfrak{d}(\cdot) d L(\mu)=L(\mu \times \mu)(\Gamma)=\int_{Y} \mathfrak{d}(\cdot) d L(\mu)=r \cdot L(\mu)(Y)
$$

So, $L(\mu)(X)=L(\mu)(Y)$ and $\mathscr{M}$ must saturate $Y$ as well because internal injective functions are obviously measure preserving.

\section{THE EXISTENCE OF PARTIAL MATCHINGS OF A $\Pi_{1}^{0}(\kappa)$ GRAPH CONTAINING A GIVEN SET IN $Y$}

So far we have examined the question of when a certain graph possesses a matching saturating some amount of vertices in $X$. Now, we shall give a result concerning the existence of a matching saturating sets in $Y$. 
Proposition 4.1. Let $X$ and $Y$ be Loeb measurable sets of finite measure and let $\Gamma \subseteq X \times Y$ be a $\Pi_{1}^{0}(\kappa)$ graph. Let $M \subseteq Y$ be Loeb measurable. Then, the following two conditions are equivalent:

(i) there exists an internal matching $\mathscr{M}$ in $\Gamma$ saturating almost all vertices in $M$,

(ii) $L(\mu)(\Gamma(A) \cap M) \geq L(\mu)(A)+L(\mu)(M)-L(\mu)(X)$, for every internal $A \subseteq X$.

If $M$ is a $\Pi_{1}^{0}(\kappa)$ set then (i) is equivalent to

(iii) $L(\mu)(\Gamma(A) \cap M) \geq L(\mu)\left(A \cap \Gamma^{-1}(M)\right)-L(\mu)\left(\Gamma^{-1}(M)\right)+L(\mu)(M)$, for every internal $A \subseteq X$.

Proof. (iii) $\Rightarrow$ (i) Let

$$
\Delta=\Gamma \cap\left(\Gamma^{-1}(M) \times M\right) .
$$

From the fact that $M$ is a $\Pi_{1}^{0}(\kappa)$ set and Lemma $1.2, \Delta$ is a $\Pi_{1}^{0}(\kappa)$ graph. We will show that $\Delta$ has an internal matching of measure defect $\leq r$ in $\Gamma^{-1}(M)$, where $r=L(\mu)\left(\Gamma^{-1}(M)\right)-L(\mu)(M)$, by showing that $\Delta$ satisfies the defect form of Hall's condition in $\Gamma^{-1}(M)$. First, if in (iii) we put $A=\varnothing$ we get

$$
0 \geq 0-L(\mu)\left(\Gamma^{-1}(M)\right)+L(\mu)(M),
$$

i.e.

$$
L(\mu)\left(\Gamma^{-1}(M)\right) \geq L(\mu)(M) .
$$

Therefore,

$$
0 \leq r \leq L(\mu)\left(\Gamma^{-1}(M)\right) .
$$

Now, let $A \subseteq \Gamma^{-1}(M)$ be internal. Then, $\Delta(A)=\Gamma(A) \cap M$ and by (iii)

$$
L(\mu)(\Delta(A)) \geq L(\mu)(A)-r .
$$

So, by the defect form of Hall's theorem (Proposition 2.2), $\Delta$ possesses an internal matching $\mathscr{M}$ of measure defect $\leq r$, which is at the same time a matching of the graph $\Gamma$. So,

$$
L(\mu)(\operatorname{dom}(\mathscr{M})) \geq L(\mu)\left(\Gamma^{-1}(M)\right)-r=L(\mu)(M),
$$

and thus, $L(\mu)(\operatorname{ran}(\mathscr{M}))=L(\mu)(M)$, which proves that $\mathscr{M}$ saturates almost all elements of $M$.

(i) $\Rightarrow$ (iii) In the terms of the proof of (iii) $\Rightarrow$ (i), if $\mathscr{M}$ is a matching of $\Gamma$ saturating almost all $M$ then $\Delta$ has a matching of measure defect at most $L(\mu)\left(\Gamma^{-1}(M)\right)-L(\mu)(M)$, so, by the defect form of Hall's theorem we have

$$
L(\mu)(\Delta(A)) \geq L(\mu)(A)-r,
$$

for every internal $A \subseteq \Gamma^{-1}(M)$. By Lemma 2.1 we have that the above condition is actually valid for all Loeb measurable subsets of $\Gamma^{-1}(M)$ when $L(\mu)$ is replaced by the inner measure $L(\mu)_{i}$. In other words,

$$
L(\mu)_{i}(\Delta(N)) \geq L(\mu)(N)-r,
$$

for every Loeb measure $N \subseteq \Gamma^{-1}(M)$. Now, recall that $\Gamma^{-1}(M)$ is, by Lemma 1.2, a $\Pi_{1}^{0}(\kappa)$ set, and thus, measurable by Lemma 1.1. Let $A \subseteq X$ be an internal subset of $X$. Then, 


$$
\begin{aligned}
L(\mu)(\Gamma(A) \cap M) & =L(\mu)\left[\Gamma\left(A \cap \Gamma^{-1}(M)\right)\right]=L(\mu)\left[\Delta\left(A \cap \Gamma^{-1}(M)\right)\right] \\
& =L(\mu)_{i}\left[\Delta\left(A \cap \Gamma^{-1}(M)\right)\right] \geq L(\mu)\left(A \cap \Gamma^{-1}(M)\right)-r \\
& =L(\mu)\left(A \cap \Gamma^{-1}(M)\right)-\left[L(\mu)\left(\Gamma^{-1}(M)\right)-L(\mu)(M)\right] \\
& =L(\mu)\left(A \cap \Gamma^{-1}(M)\right)-L(\mu)\left(\Gamma^{-1}(M)\right) L(\mu)(M),
\end{aligned}
$$

and (iii) is proved.

(ii) $\Leftrightarrow$ (i) Suppose first that $M$ is an internal set. If we keep the same notation as in the proof of (ii) $\Rightarrow$ (i) and (i) $\Rightarrow$ (ii) and having in mind these proofs, it becomes clear that $\Gamma$ has an internal matching saturating almost all elements of $M$ if and only if the graph $\Delta$ has an internal matching of measure defect

$\left[L(\mu)\left(\Gamma^{-1}(M)\right)-L(\mu)(M)\right]+\left[L(\mu)(X)-L(\mu)\left(\Gamma^{-1}(M)\right)\right]=L(\mu)(X)-L(\mu)(M)$,

in $X$. This is, in turn, equivalent to (ii) because for every internal subset $A$ of $X$ we have $\Delta(A)=\Gamma(A) \cap M$.

Now, let $M$ be arbitrary Loeb measurable set. Let $B$ be an internal set such that $L(\mu)(B \Delta M)=0$. To make $B$ a subset of $Y$ we put $C=B \cap \operatorname{ran}(\Gamma)$. Then, $C$ is a $\Pi_{1}^{0}(\kappa)$ set and $L(\mu)(C \Delta M)=0$. Now, $\Gamma$ has an internal matching saturating almost all $M$ if and only if $\Gamma$ has an internal matching saturating almost all $C$. By the first part of the proof this is true if and only if

$$
L(\mu)(\Gamma(A) \cap C) \geq L(\mu)(A)+L(\mu)(C)-L(\mu)(X) .
$$

But, $L(\mu)(\Gamma(A) \cap C)=L(\mu)(\Gamma(A) \cap M)), L(\mu)(C)=L(\mu)(M)$ and we are done.

\section{THE EXISTENCE OF A FUll MATCHING of A $\Pi_{1}^{0}(\kappa)$ GRAPH CONTAINING A GIVEN SET IN $Y$}

The following proposition answers the question of when a $\Pi_{1}^{0}(\kappa)$ graph possesses an internal matching which saturates almost all elements of a given Loeb measurable set $M$ in $Y$ and almost all elements of the set $X$. It is obvious that a necessary condition for the existence of such a matching is that a graph possesses an internal matching saturating almost all of $X$ and, separately, an internal matching saturating almost all of $M$. It is surprising that these two conditions are also sufficient.

Proposition 5.1. Let $X$ and $Y$ be Loeb measurable sets of finite measure and let $\Gamma \subseteq X \times Y$ be a $\Pi_{1}^{0}(\kappa)$ graph. Let $M \subseteq Y$ be Loeb measurable. Then, there exists an internal matching of $\Gamma$ saturating almost all elements of $X \cup M$ if and only if the following two conditions are satisfied:

(i) $\Gamma$ has an internal matching saturating almost all of $X$;

(ii) $\Gamma$ has an internal matching saturating almost all of $M$.

Proof. It is clear that the conditions (i) and (ii) are necessary. Assume (i) and (ii). We can suppose that $Y=\operatorname{ran}(\Gamma)$. By condition (i) we have $L(\mu)(Y) \geq$ $L(\mu)(X)$. Let $Z$ be an internal set of measure $L(\mu)(Y)-L(\mu)(X)$, disjoint from $X$, i.e.,

$$
L(\mu)(Z)=L(\mu)(Y)-L(\mu)(X), \quad X \cap Z=\varnothing .
$$


Let $B_{n} \quad(n \in \omega)$ be an increasing sequence of internal sets such that $\bigcup_{n} B_{n}$ approximates $M$ from within, i.e.,

$$
B_{n} \subseteq B_{n+1}, \quad L(\mu)\left(M \backslash \bigcup_{n} B_{n}\right)=0 .
$$

We shall now define a new graph $\Delta \subseteq(X \cup Z) \times Y$ by

$$
\Delta=\Gamma \cup\left(Z \times \operatorname{ran}(\Gamma) \backslash\left(\bigcup_{n} B_{n}\right)\right) .
$$

It is clear that $\Delta$ is a $\Pi_{1}^{0}(\kappa)$ graph. Now, note that $\Gamma$ has an internal matching saturating almost all of $M$ and almost all of $X$ if and only if $\Gamma$ has an internal matching saturating almost all of $\bigcup_{n} B_{n}$ and almost all of $X$. This is equivalent to the statement that $\Delta$ has an internal matching $\mathscr{K}$ saturating almost all elements of $X \cup Z$ (just take $\mathscr{M}=\mathscr{K} \backslash(Z \times \Omega)$ for desired matching in $\Gamma$ ). So, we have to verify if the Hall's condition is satisfied for the graph $\Delta$.

Let $A \subseteq X \cup Z$ be internal. Then, $A$ can be decomposed as $A=B \cup C$, where $B$ and $C$ are internal and $B \subseteq X$ and $C \subseteq Z$. Let $T=\bigcup_{n} B_{n}$.

If $C \neq \varnothing$ then

$$
\Delta(A)=\Delta(B \cup C)=(\Gamma(B) \cap T) \cup(\operatorname{ran}(\Gamma)) \backslash T,
$$

so,

$$
\begin{aligned}
L(\mu)(\Delta(A)) & =L(\mu)(\Gamma(B) \cap T)+L(\mu)(\operatorname{ran} \Gamma)-L(\mu)(T) \\
& =L(\mu)(\Gamma(B) \cap T)+L(\mu)(Y)-L(\mu)(M) .
\end{aligned}
$$

Now, by the second part of Proposition 4.3 and by (ii) we have

$$
\begin{aligned}
L(\mu)(\Delta(A)) & \geq[L(\mu)(B)+L(\mu)(M)-L(\mu)(X)]+[L(\mu)(Y)-L(\mu)(M)] \\
& =L(\mu)(B)+L(\mu)(Y)-L(\mu)(X)=L(\mu)(B)+L(\mu)(Z) \\
& \geq L(\mu)(B)+L(\mu)(C)=L(\mu)(A) .
\end{aligned}
$$

If $C=\varnothing$ then $\Delta(A)=\Gamma(A)$ and by (i) and Hall's theorem $L(\mu)(\Delta(A)) \geq$ $L(\mu)(A)$.

We draw the following corollary of the previous proposition using the form of Hall's condition proved before.

Corollary 5.2. Let $X$ and $Y$ be Loeb measurable sets of finite measure and let $\Gamma \subseteq X \times Y$ be a $\Pi_{1}^{0}(\kappa)$ graph. Let $M \subseteq Y$ be Loeb measurable. Then, $\Gamma$ has an internal matching saturating almost all elements of $X$ and $M$ at the same time if and only if

(i) $L(\mu)(\Gamma(A)) \geq L(\mu)(A)$, and

(ii) $L(\mu)(M \backslash \Gamma(A)) \leq L(\mu)(X \backslash A)$,

for every internal $A \subseteq \bar{X}$.

Proof. Condition (i) is equivalent to the existence of an internal matching of $\Gamma$ saturating almost all of $X$ and we only have to prove that (ii) is equivalent to the existence of an internal matching of $\Gamma$ saturating almost all of $M$. 
We first take an internal $B$ with $L(\mu)(B \Delta M)=0$. Then $\Gamma$ has an internal matching saturating almost all of $M$ if and only if the graph $\Gamma^{-1} \cap(\Omega \times B)$, which is considered to be defined on $B$, has an internal matching saturating almost all elements of $B$. By Proposition 1.3 this is equivalent to

$$
L(\mu)\left[B \backslash\left(\Gamma^{-1} \cap(\Omega \times B)\right)^{-1}(A)\right] \leq L(\mu)(X \backslash A),
$$

for every internal $A \subseteq X$. But,

$$
B \backslash\left(\Gamma^{-1} \cap(\Omega \times B)\right)^{-1}(A)=B \backslash[(\Gamma \cap(\Omega \times B))(A)]=B \backslash \Gamma(A) \cap B=B \backslash \Gamma(A),
$$

and $L(\mu)(B \backslash \Gamma(A))=L(\mu)(M \backslash \Gamma(A))$, and we are done.

\section{THE EXISTENCE OF A MATCHING OF A $\Pi_{1}^{0}(\kappa)$ GRAPH CONTAINING} A GIVEN SET IN $Y$ AND HAVING A PRESCRIBED MEASURE DEFECT

We shall now give the defect form of Proposition 5.1. If we are given a Loeb measurable set $M$ in $Y$ and a $\Pi_{1}^{0}(\kappa)$ graph in $X \times Y$, we want to determine when there exists an internal matching of $\Gamma$ which saturates $M$ a.e. and such that the measure of the set of saturated vertices in $X$ is at least $r$, where $r$ is a given real number. As one can expect, the answer is similar to one given in Proposition 5.1.

Proposition 6.1. Let $X$ and $Y$ be Loeb measurable sets of finite measure and let $\Gamma \subseteq X \times Y$ be a $\Pi_{1}^{0}(\kappa)$ graph. Let $M \subseteq Y$ be a Loeb measurable set and $r$ a real number satisfying

$$
L(\mu)(M) \leq r \leq L(\mu)(X) .
$$

Then, there exists an internal matching of $\Gamma$ saturating almost all elements of $M$ and such that the measure of saturated vertices in $X$ is at least $r$ if and only if the following two conditions are satisfied:

(i) $\Gamma$ has an internal matching with defect $\leq L(\mu)(X)-r$ in $X$;

(ii) $\Gamma$ has an internal matching saturating almost all of $M$.

Proof. The proof is similar to one given for the defect form of Hall's theorem. The only difference is that here, instead of Hall's theorem, we use Proposition 5.1 .

It is clear that the conditions (i) and (ii) are necessary. To prove the converse let $Z$ be an internal set of measure $L(\mu)(X)-r$ and disjoint from $Y$. Let $\Delta \subseteq X \times(Y \cup Z)$ be a new graph defined as

$$
\Delta=\Gamma \cup(\operatorname{dom}(\Gamma) \times Z) .
$$

It is clear that $\Delta$ is a $\Pi_{1}^{0}(\kappa)$ set. By the condition (i) of the proposition, $\Delta$ possesses an internal matching saturating almost all of $X$. By condition (ii), $\Delta$ has an internal matching saturating almost all of $M$. Thus, by Proposition 5.1, $\Delta$ has an internal matching $\mathscr{K}$ saturating almost all elements of $X \cup M$. If we define $\mathscr{M}=\mathscr{K} \backslash(\Omega \times Z)$, then $\mathscr{M}$ would be an internal matching of $\Gamma$ saturating almost all of $M$. Moreover,

$$
L(\mu)(\operatorname{dom}(M)) \geq L(\mu)(X)-(L(\mu)(X)-r)=r,
$$

and $\mathscr{M}$ saturates a set in $X$ which measure is at least $r$. 


\section{THE EXISTENCE OF A LINK OF TWO SETS IN A $\Pi_{1}^{0}(\kappa)$ GRAPH}

Given two Loeb measurable sets $M$ and $N$ in $X$ and $Y$ respectively, and a $\Pi_{1}^{0}(\kappa)$ graph in $X \times Y$, one can ask if the similar conditions to one given in the Proposition 6.1 would lead to an internal matching, called a link of $M$ and $N$, a.e. saturating both $M$ and $N$. The following proposition answers that question positively.

Proposition 7.1. Let $X$ and $Y$ be Loeb measurable sets of finite measure and let $\Gamma \subseteq X \times Y$ be a $\Pi_{1}^{0}(\kappa)$ graph. Let $M \subseteq X$ and $N \subseteq Y$ be Loeb measurable.

Then, there exists an internal matching $\mathscr{M}$ of $\Gamma$ and internal sets $A_{1}$ containing $M$ almost surely and $A_{2}$ containing $N$ almost surely and such that $A_{1}$ and $A_{2}$ are saturated by $\mathscr{M}$ if and only if the following two conditions are satisfied:

(i) $\Gamma$ has an internal matching saturating almost all of $M$;

(ii) $\Gamma$ has an internal matching saturating almost all of $N$.

Proof. The conditions (i) and (ii) are obviously necessary. Let us prove their sufficiency.

We can suppose that $\operatorname{dom}(\Gamma)=X$ and $\operatorname{ran}(\Gamma)=Y$. Take a $\Sigma_{1}^{0}$ subsets $T$ and $S$ of $M$ and $N$ respectively, $T \subseteq M, S \subseteq N$, such that

$$
L(\mu)(M \backslash T)=L(\mu)(N \backslash S)=0 .
$$

Let $F$ be an internal set of measure $L(\mu)(X)$ disjoint from $Y$, and $E$ an internal set of measure $L(\mu)(Y)$ disjoint from $X$

$$
\begin{aligned}
& L(\mu)(F)=L(\mu)(X), \quad F \cap Y=\varnothing ; \\
& L(\mu)(E)=L(\mu)(Y), \quad E \cap X=\varnothing \text {. }
\end{aligned}
$$

Let us define a new graph $\Delta \subseteq(X \cup E) \times(Y \cup F)$ by

$$
\Delta=\Gamma \cup(E \times(Y \backslash S)) \cup((X \backslash T) \times F) \cup(E \times F) .
$$

As before, it is easy to see that $\Delta$ is a $\Pi_{1}^{0}(\kappa)$ graph. Let us prove that $\Delta$ satisfies Hall's condition. Let $A \subseteq X \cup E$ be internal set. Then, $A$ can be partitioned into sets $B, C$ and $D, A=B \cup C \cup D$, such that $D \subseteq E$ is internal, $B \subseteq T$ is $\Sigma_{1}^{0}$ and $C \subseteq X \backslash T$ is a $\Pi_{1}^{0}(\kappa)$ set.

We have to check seven cases.

I. $B=C=\varnothing, D \neq \varnothing$. We have

$$
\begin{aligned}
L(\mu)(\Delta(A)) & =L(\mu)(\Delta(D)) \geq L(\mu)(Y)-L(\mu)(S) \geq L(\mu)(Y) \\
& \geq L(\mu)(D)=L(\mu)(A),
\end{aligned}
$$

because $D \subseteq E$ and $L(\mu)(E)=L(\mu)(Y)$.

II. $B=D=\varnothing, C \neq \varnothing$. We have

$$
\begin{aligned}
L(\mu)(\Delta(A)) & =L(\mu)(\Delta(C)) \geq L(\mu)(F)=L(\mu)(X) \\
& \geq L(\mu)(X)-L(\mu)(T) \geq L(\mu)(C)=L(\mu)(A),
\end{aligned}
$$

because $C \subseteq X \backslash T$. 
III. $C=D=\varnothing, B \neq \varnothing$. We have

$$
\begin{aligned}
L(\mu)(\Delta(A)) & =L(\mu)(\Delta(B))=L(\mu)(\Gamma(B)) \\
& \geq L(\mu)(B)=L(\mu)(A) .
\end{aligned}
$$

To justify the inequality $L(\mu)(\Gamma(B)) \geq L(\mu)(B)$ note that, by (i), $\Gamma$ satisfies Hall's condition on a $\Pi_{1}^{0}$ set containing $M$ with $M$ being of full measure in it. Moreover, as $B$ is a $\Sigma_{1}^{0}$ set, $\Gamma(B)$ is measurable and the result follows by Lemma 2.1 .

IV. $B=\varnothing, C \neq \varnothing, D \neq \varnothing$. Note first that $C$ and $D$ are both internal. Then,

$$
\begin{aligned}
L(\mu)(\Delta(A)) & =L(\mu)(\Delta(C \cup D)) \\
& =L(\mu)(F)+L(\mu)(\Gamma(C) \cap S)+L(\mu)(Y)-L(\mu)(S) \\
& =L(\mu)(X)+L(\mu)(\Gamma(C) \cap S)+L(\mu)(Y)-L(\mu)(S) .
\end{aligned}
$$

Now by (ii) and by the construction of the set $S$, we have that $\Gamma$ has an internal matching saturating $S$ a.e. so, by the equivalence (iii) Proposition 4.3,

$$
L(\mu)(\Gamma(C) \cap S) \geq L(\mu)(C)+L(\mu)(S)-L(\mu) X) .
$$

Finally, we have

$$
\begin{aligned}
L(\mu)(\Delta(A)) & \geq L(\mu)(C)+L(\mu)(Y) \geq L(\mu)(C)+L(\mu) D) \\
& =L(\mu)(C \cup D)=L(\mu)(A) .
\end{aligned}
$$

V. $B \neq \varnothing, C=\varnothing, D \neq \varnothing$. Note that, again, $B$ and $D$ are internal. We have

$$
\begin{aligned}
L(\mu)(\Delta(A))= & L(\mu)(\Delta(B \cup D)) \\
= & L(\mu)(\Gamma(B) \cap S)+L(\mu)(Y)-L(\mu)(S)+L(\mu)(F) \\
\geq & {[L(\mu)(B)+L(\mu)(S)-L(\mu)(X)] } \\
& +L(\mu)(Y)-L(\mu)(S)+L(M)(X) \\
= & L(M)(B)+L(\mu)(Y) \geq L(\mu)(B)+L(\mu)(D)=L(\mu)(A),
\end{aligned}
$$

where we use similar arguments as in case IV.

VI. $B \neq \varnothing, C \neq \varnothing, D=\varnothing$. This case is trivial because,

$$
\begin{aligned}
L(\mu)(\Delta(A)) & =L(\mu)(\Delta(B \cup C)) \geq L(\mu)(\Delta(C)) \\
& =L(\mu)(F)=L(\mu)(X) \geq L(\mu)(B \cup C)=L(\mu)(A) .
\end{aligned}
$$

VII. $B \neq \varnothing, C \neq \varnothing, D \neq \varnothing$. Note that $B \cup C$ is internal. We have

$$
\begin{aligned}
L(\mu)(\Delta(A))= & L(\mu)(\Gamma(B \cup C) \cap C)+L(\mu)(F)+L(\mu)(Y)-L(\mu)(S) \\
\geq & {[L(\mu)(B \cup C)+L(\mu)(S)-L(\mu)(X)] } \\
& +L(\mu)(X)+L(\mu)(Y)-L(\mu)(S) \\
= & L(\mu)(B \cup C)+L(\mu)(Y) \geq L(\mu)(B \cup C)+L(\mu)(D)=L(\mu)(A),
\end{aligned}
$$

where we use similar arguments as in IV.

So, Hall's condition is satisfied for $\Delta$. Therefore, $\Delta$ has an internal matching $\mathscr{K}$ saturating almost all $X \cup E$ and thus, almost all $Y \cup F$. Take $\mathscr{M}=$ 
$\mathscr{K} \backslash[(E \times \Omega) \cup(\Omega \times F)]$. Then $\mathscr{M}$ is an internal matching of $\Gamma$ with $\operatorname{dom}(\mathscr{M}) \supseteq$ $T$ a.e. and $\operatorname{ran}(\mathscr{M}) \supseteq N$ a.e. So, we may take $A_{1}=\operatorname{dom}(\mathscr{M})$ and $A_{2}=$ $\operatorname{ran}(\mathscr{M})$.

\section{THE EXISTENCE OF A LINK OF TWO SETS IN A $\Pi_{1}^{0}(\kappa)$ GRAPH WITH PRESCRIBED MEASURE DEFECTS}

One can give a defect form of Proposition 7.1, that is if $\Gamma$ possesses only an internal matching of some measure defect in $M$ and an internal matching of some measure defect in $N$, then, there exist an internal matching of $\Gamma$ of prescribed measure defects in $M$ and $N$.

Proposition 8.1. Let $X$ and $Y$ be Loeb measurable sets of finite measure and let $\Gamma \subseteq X \times Y$ be a $\Pi_{1}^{0}(\kappa)$ graph. Let $M \subseteq X$ and $N \subseteq Y$ be Loeb measurable.

Then, given real numbers $r$ and $s$, there exists an internal matching $\mathscr{M}$ of $\Gamma$ and internal sets $A_{1}$ and $A_{2}$ a.e. saturated by $\mathscr{M}$ and such that

$$
L(\mu)\left(A_{1} \cap M\right) \geq L(\mu)(M)-r
$$

and

$$
L(\mu)\left(A_{2} \cap N\right) \geq L(\mu)(N)-s
$$

if and only if the following two conditions are satisfied:

(i) $\Gamma$ has an internal matching of defect $\leq r$ in $M$;

(ii) $\Gamma$ has an internal matching of defect $\leq s$ in $N$.

Proof. The above conditions are obviously necessary. Let (i) and (ii) be true. Let $T \supseteq M$ and $S \supseteq N$ be $\Pi_{1}^{0}$ sets with $L(\mu)(T \backslash M)=L(\mu)(S \backslash N)=0$ and let $E$ and $F$ be internal sets with $L(\mu)(E)=s, L(\mu)(F)=r, F \cap Y=E \cap X=\varnothing$. We define a new graph $\Delta$ as $\Delta=\Gamma \cup(T \times F) \cup(E \times S)$. It is clear that $\Delta$ is a $\Pi_{1}^{0}(\kappa)$ graph.

Now, it is plain that $\Delta$ has an internal matching saturating almost all $S$ and an internal matching saturating almost all $T$, which at the same time saturates almost all $N$ and almost all $M$. By Proposition 7.1, there exists an internal matching $\mathscr{K}$ in $\Delta$ saturating almost all $M$ and $N$ at the same time. Our desired matching is then,

$$
\mathscr{M}=\mathscr{K} \backslash[(E \times \Omega) \cup(\Omega \times F)],
$$

and desired internal sets

$$
A_{1}=\operatorname{dom}(\mathscr{M}), \quad A_{2}=\operatorname{ran}(\mathscr{M})
$$

\section{THE EXISTENCE OF MATCHINGS OF A $\Pi_{1}^{0}(\kappa)$ GRAPH WITH PRESCRIBED DEFECTS AND DISJOINT RANGES}

The following result is an inverse situation to one considered in Proposition 3.1. In Proposition 3.1 we asked for $n$ internal matchings with disjoint domains and now, we ask for $n$ internal matchings of prescribed global measure defect whose ranges are mutually disjoint. For a real number $r$ we set $r^{+}=\max \{r, 0\}$. 
Proposition 9.1. Let $X$ and $Y$ be Loeb measurable sets $X$ being of finite measure. Let $\Gamma \subseteq X \times Y$ be a $\Pi_{1}^{0}(\kappa)$ graph and let $r_{1}, \ldots, r_{n}$ be real numbers with

$$
0 \leq r_{i} \leq L(\mu)(X) \quad(i=1, \ldots, n) .
$$

Then, $\Gamma$ possesses $n$ internal matchings $\mathscr{M}_{i}(i=1, \ldots, n)$ such that the measure defect $\delta\left(\mathscr{M}_{i}\right)$ of $\mathscr{M}_{i}$ is less than or equal to $r_{i}$, and such that $\operatorname{ran}\left(\mathscr{M}_{i}\right)$ $(i=1, \ldots, n)$ are mutually disjoint, if and only if

$$
L(\mu)(\Gamma(A)) \geq \sum_{i=1}^{n}\left(L(\mu)(A)-r_{i}\right)^{+},
$$

for every internal $A \subseteq X$.

Proof. $\Rightarrow:$ Suppose that we have internal matchings $\mathscr{M}_{i}(i=1, \ldots, n)$ having the prescribed properties. That is, the ranges of $\mathscr{M}_{i}(i=1, \ldots, n)$ are disjoint and $\mathscr{M}_{i}$ has measure defect $\leq r_{i}$. In view of the defect form of Hall's theorem (Proposition 2.2) we have

$$
L(\mu)\left(\mathscr{M}_{i}(A)\right) \geq L(\mu)(A)-r_{i},
$$

for every internal $A \subseteq X$.

Now, let $A \subseteq X$ be an internal set. We have $\Gamma \supseteq \bigcup_{i=1}^{n} \mathscr{M}_{i}$ so, taking in account that the ranges of $\mathscr{M}_{i}(i=1, \ldots, n)$ are disjoint,

$$
L(\mu)(\Gamma(A)) \geq \sum_{i=1}^{n} L(\mu)\left(\mathscr{M}_{i}(A)\right) \geq \sum_{i=1}^{n}\left(L(\mu)(A)-r_{i}\right)^{+} .
$$

$\Leftarrow$ : Let $F_{i} \quad(i=1, \ldots, n)$ be disjoint internal sets with $L(\mu)\left(F_{i}\right)=r_{i}$ $(i=1, \ldots, n)$. Moreover, we suppose that $F_{i} \cap Y=\varnothing$. Let us introduce a counting measure $\nu$ in the set $\{1, \ldots, n\}$ as $\nu(A)=|A|$.

The product measure $L(\mu \times \nu)$ in $\Omega \times\{1, \ldots, n\}$ is nothing but the Loeb measure of the internal counting measure obtained by dividing internal subsets of $\Omega \times\{1, \ldots, n\}$ by $H$.

Let

$$
\Delta \subseteq X \times\{1, \ldots, n\} \times Y,
$$

be a graph defined as

$$
(x, k, y) \in \Delta \text { if and only if }(x, y) \in \Gamma \text { or }(x, y) \in \Omega \times F_{k} .
$$

The graph $\Delta$ is constructed by making $n$ copies of the graph $\Gamma$ and adding the set $\Omega \times F_{i}$ to $i$ th copy. It is easy to see that $\Delta$ is $\Pi_{1}^{0}(\kappa)$. Moreover, $\Delta$ satisfies Hall's condition when the domain of $\Delta$ is considered to be the set $X \times\{1, \ldots, n\}$. Indeed, let $A \subseteq X \times\{1, \ldots, n\}$ be an internal set and let

$$
\pi: X \times\{1, \ldots, n\} \rightarrow X,
$$

be the projection map. Then,

$$
\Delta(A)=\left(\bigcup_{i=1}^{m} F_{k_{i}}\right) \cup \Gamma(\pi(A)),
$$

where the integers $k_{1}, \ldots, k_{m} \in\{1, \ldots, n\}$ are all the integers satisfying $A \cap$ $X \times\left\{k_{i}\right\} \neq \varnothing$. Therefore, we have 


$$
\begin{aligned}
L(\mu)(\Delta(A)) & \geq \sum_{i=1}^{m} r_{k_{i}}+\sum_{i=1}^{n}\left(L(\mu)(\pi(A))-r_{i}\right)^{+} \\
& \geq \sum_{i=1}^{m} r_{k_{i}}+\sum_{i=1}^{m}\left(L(\mu)(\pi(A))-r_{i}\right)^{+} \\
& \geq \sum_{i=1}^{m} r_{k_{i}}+\sum_{i=1}^{m}\left(L(\mu)(\pi(A))-r_{i}\right) \\
& =m \cdot L(\mu)(\pi(A)) \geq L(\mu)(A),
\end{aligned}
$$

because $A \subseteq \bigcup_{i=1}^{m} \pi(A) \times\left\{k_{i}\right\}$.

So, $\Delta$ satisfies Hall's condition and therefore, possesses an internal matching $\mathscr{M}$ saturating almost all of $X \times\{1, \ldots, n\}$. Define

$$
\mathscr{K}_{k}=\mathscr{M} \cap\left(\Omega \times\{k\} \times\left(\Omega \backslash F_{k}\right)\right),
$$

and finally,

$$
\mathscr{M}_{k}=\operatorname{pr}\left(\mathscr{K}_{k}\right)
$$

where $\operatorname{pr}: \Omega \times\{1, \ldots, n\} \times \Omega \rightarrow \Omega \times \Omega$ is the projection map.

\section{HALL'S THEOREM AND RELATED RESULTS FOR $\Sigma_{1}^{0}(\kappa)$ MONOTONE GRAPHS}

As we have seen so far, the Transversal Theory for $\Pi_{1}^{0}(\kappa)$ graphs develops in the same way as the standard Transversal Theory. We may say that, in this case, any result in the standard Transversal Theory has its counterpart in the hyperfinite case, where the necessary terminology is adjusted to measure case, i.e. "saturated" is replaced by "a.e. saturated", "the number of elements" is substituted by "measure" and so on.

Unfortunately, the same is not true for arbitrary graphs. When the graph $\Gamma$ is a $\Sigma_{1}^{0}(\kappa)$ monotone graph or just a $\Sigma_{1}^{0}$ graph (which, of course, implies that it is a $\Sigma_{1}^{0}$ monotone graph, as well) and satisfies Hall's condition, one cannot claim the existence of a matching saturating almost all of the domain of the graph. However, the existence of such a matching can be claimed if one allows arbitrary small measure defects in the conclusion. That is, for every $\varepsilon>0$ the graph in question will possess an internal matching of measure defect $\leq \varepsilon$. This gives rise to an approximation version of Hall's theorem which, in the same manner as Hall's theorem for $\Pi_{1}^{0}(\kappa)$ graphs, yields an approximation version of the Hyperfinite Transversal Theory. In the conclusions of the formulations of the analogous statements to ones given so far for the case of $\Pi_{1}^{0}(\kappa)$ graphs, the phrase "... an internal matching saturating almost all domain of ..." should be replaced by "... an internal matching of measure defect $\leq \ldots$ " to get a true statement.

We start with the approximation form of Hall's theorem. Recall that a $\Sigma_{1}^{0}(\kappa)$ monotone graph is an increasing union of internal graphs, i.e. a graph of the form

$$
\Gamma=\bigcup_{\alpha<\kappa} \Gamma_{\alpha}, \quad \Gamma_{\alpha} \subseteq \Gamma_{\beta} \quad(\alpha<\beta)
$$


Proposition 10.1. Let $X$ and $Y$ be Loeb measurable of finite measure. Let $\Gamma \subseteq X \times Y$ be a $\Sigma_{1}^{0}(\kappa)$ monotone graph. Then, for every $\varepsilon>0$ there exists an internal matching $\mathscr{M}_{\varepsilon} \subseteq \Gamma$ of measure defect $\leq \varepsilon$ in $X$ if and only if

$$
L(\mu)(\Gamma(A)) \geq L(\mu)(A),
$$

for every internal $A \subseteq X$.

Proof. $\Leftarrow$ : Suppose that Hall's condition is satisfied. Let us first assume that $X$ and $Y$ are internal. Let

$$
\Gamma=\bigcup_{\alpha<\kappa} \Gamma_{\alpha}, \quad \Gamma_{\alpha} \subseteq \Gamma_{\beta} \quad(\alpha<\beta),
$$

where $\Gamma_{\alpha}(\alpha<\kappa)$ are internal. We may assume that $\Gamma_{\alpha} \subseteq X \times Y$. Extend $\Gamma_{\alpha}$ to an internal sequence

$$
\Gamma_{\tau} \quad\left(\tau \in{ }^{*} \kappa\right) .
$$

The internal sequence $\Gamma_{\tau}$ may be taken to be increasing in $\tau$. Therefore for any $*$-ordinal $\tau$ greater than each standard $\alpha<{ }^{*} \kappa$,

$$
\Gamma_{\tau} \supseteq \bigcup_{\alpha<\kappa} \Gamma_{\alpha}=\Gamma \text {. }
$$

Now, for every $\tau \epsilon^{*} \kappa$ we can define the internal defect $d_{\tau}$ of the graph $\Gamma_{\tau}$ as

$$
d_{\tau}=\sup \left\{\frac{|A|}{H}-\frac{\left|\Gamma_{\tau}(A)\right|}{H}: A \subseteq X \text { internal }\right\} .
$$

We know that $d_{\tau} \leq 0$ or $d_{\tau} \approx 0$ for every ordinal $\tau \in^{*} \kappa$ which is greater than any standard ordinal. By saturation, for every $\varepsilon>0$ there exists an $\alpha<\kappa$ with $d_{\alpha} \leq \varepsilon$. By the defect version of Hall's theorem, $\Gamma_{\alpha}$ and thus, $\Gamma$ possesses an internal matching saturating a set in $X$ of measure $\geq L(\mu)(X)-\varepsilon$. The claim is proved.

Now, let $X$ and $Y$ be arbitrary Loeb measurable sets. As $X$ is of finite measure, there exists an internal set $Z$ with $L(\mu)(X \Delta Z)=0$. Let $\Delta \subseteq Z \times \Omega$ be defined as $\Delta=(Z \times \Omega) \cap \Gamma$. Then, $\Delta$ is a $\Sigma_{1}^{0}(\kappa)$ monotone graph and $\Gamma$ has an internal matching of measure defect $\leq \varepsilon$ if and only if $\Delta$ has an internal matching of measure defect $\leq \varepsilon$. It remains only to check that $\Delta$ satisfies Hall's condition. For $A \subseteq Z$ internal we have

$$
\left.L(\mu)(A)=L(\mu)(A \cap X) \leq L(\mu)_{i} \Gamma(A \cap X)\right)=L(\mu)_{i}(\Delta(A \cap X)) \leq L(\mu)(\Delta(A)),
$$

where in the first inequality we used Lemma 2.1 .

$\Rightarrow$ : Obvious.

As we have said earlier, after an approximation version of Hall's theorem (Proposition 10.1) we may give, using similar techniques, the corresponding approximation results for defect, partition-defect or regular-graph versions of Hall's theorem. We shall list some of these results whose proofs are, after corresponding proofs in $\S \S 2-9$ clear.

Proposition 10.2. Let $X$ and $Y$ be Loeb measurable sets of finite measure and $r$ a real number. Let $\Gamma \subseteq X \times Y$, be a $\Sigma_{1}^{0}(\kappa)$ monotone graph. Then, the following two conditions are equivalent: 
(i) for every $\varepsilon>0, \Gamma$ has an internal matching such that the measure of nonsaturated vertices in $X$ is less than or equal to $r+\varepsilon$;

(ii)

$$
L(\mu)(\Gamma(A)) \geq L(\mu)(A)-r
$$

for every internal subset $A$ of $X$.

Proof. $\Leftarrow:$ As in the proof of Proposition 2.2 we shall augment the set of vertices $Y$ by a suitable set and get a new graph which satisfies Hall's condition. The only thing we should take care about is that the new graph should also be $\Sigma_{1}^{0}(\kappa)$ monotone. Let $D=\bigcup_{\alpha<\kappa} D_{\alpha}, D_{\alpha} \subseteq D_{\beta} \quad(\alpha<\beta)$ be a $\Sigma_{1}^{0}(\kappa)$ monotone set of measure $r$ with $D \cap Y=\varnothing$. Let $A_{\alpha} \subseteq X(\alpha<\kappa)$ be an increasing sequence of internal sets such that $L(\mu)\left(\bigcup_{\alpha<\kappa} A_{\alpha}\right)=L(\mu)(X)$. Let

$$
\Delta \subseteq X \times(Y \cup D)
$$

be a new graph defined as

$$
\Delta=\bigcup_{\alpha<\kappa}\left(\Gamma_{\alpha} \cup\left(A_{\alpha} \times D_{\alpha}\right)\right)
$$

where, as usual, $\Gamma=\bigcup_{\alpha<\kappa} \Gamma_{\alpha}, \Gamma_{\alpha} \subseteq \Gamma_{\beta} \quad(\alpha<\beta), \Gamma_{\alpha}$ internal. It is clear that $\Delta$ is $\Sigma_{1}^{0}(\kappa)$ monotone graph.

All we have to show now is that $\Delta$ satisfies Hall's condition. But, this is clear because if $A \subseteq \bigcup_{\alpha<\kappa} A_{\alpha}$ is an internal subset of $A$ then $A$ is an internal subset of $X$ as well and $\Delta(A)=\Gamma(A) \cup D$.

$\Rightarrow$ : Trivial.

Corollary 10.3. Let $X$ and $Y$ be Loeb measurable sets of finite measure and let $\Gamma \subseteq X \times Y$ be a $\Sigma_{1}^{0}(\kappa)$ monotone graph. Then,

$$
\delta(\Gamma)=\sup \{L(\mu)(A)-L(\mu)(\Gamma(A)): A \subseteq X \text { internal }\},
$$

where $\delta(\Gamma)$ is as usual the measure defect of the graph $\Gamma$.

The following propositions and corollaries correspond to Propositions 3.1 and 3.5, and Corollaries 3.6 and 3.7. The proofs, which are entirely analogous to those given before, are omitted.

Proposition 10.4. Let $X$ and $Y$ be Loeb measurable sets of finite measure. Let $\Gamma \subseteq X \times Y$ be a $\Sigma_{1}^{0}(\kappa)$ monotone graph and $r$ a real number. Then, the following two conditions are equivalent:

(i) for every $\varepsilon>0, X$ can be partitioned into $n$ Loeb measurable parts $X=\bigcup_{i=1}^{n} X_{i}$ such that there exists an internal function $\mathcal{N}$ in $\Gamma$ whose restriction to each of the members of the partition is injective and such that the measure of the set of vertices in $X$ nonsaturated by $\mathscr{N}$ is less than or equal to $r+\varepsilon$;

$$
n \cdot L(\mu)(\Gamma(A)) \geq L(\mu)(A)-r,
$$

for every internal subset $A \subseteq X$ of $X$.

Recall that $\mathfrak{d}$ is the degree function of the graph $\Gamma$.

Proposition 10.5. Let $X$ and $Y$ be Loeb measurable sets of finite measure. Let $\Gamma \subseteq X \times Y$ be a $\Sigma_{1}^{0}(\kappa)$ monotone graph. Suppose that

$$
\mathfrak{o}(\alpha) \leq r_{1} \text { a.e. on } Y \text { and } \mathfrak{d}(\alpha) \geq r_{2} \text { a.e. on } X \text {. }
$$


Then, for every $\varepsilon>0, X$ can be partitioned into $\left[r_{1} / r_{2}\right]$ Loeb measurable parts such that there exists an internal function $\mathscr{M}$ in $\Gamma$ whose restriction to each of the members of the partition is injective and such that $L(\mu)(X) \backslash \operatorname{dom}(\mathscr{M}) \leq \varepsilon$ (recall that $\left[r_{1} / r_{2}\right]$ is the least integer greater or equal to $r_{1} / r_{2}$ ).

Recall that $\mathfrak{i}(f)$ is the essentiai infimum and $\mathfrak{s}(f)$ is the essential suprememum of a Loeb measurable, real-valued function $f$.

Corollary 10.6. Let $X$ and $Y$ be Loeb measurable sets of finite measure. Let $\Gamma \subseteq X \times Y$ be a $\Sigma_{1}^{0}(\kappa)$ monotone graph and $\mathfrak{d} \mid X(\mathfrak{d} \mid Y)$ the restriction of the degree function $\mathfrak{d}$ to $X(Y)$. Suppose that

$$
\mathfrak{s}(\mathfrak{d} \mid Y) \leq \mathfrak{i}(\mathfrak{d} \mid X) .
$$

Then, for every $\varepsilon>0, \Gamma$ has an internal matching saturating a set in $X$ of measure $\geq L(\mu)(X)-\varepsilon$.

Corollary 10.7. Let $X$ and $Y$ be Loeb measurable sets of finite measure and let $\Gamma \subseteq X \times Y$ be $\Sigma_{1}^{0}(\kappa)$ monotone graph. Suppose that $\Gamma$ is a.e. $r$-regular, that is

$$
\mathfrak{d}(x)=r \text { a.e. on } X \cup Y .
$$

Then, for every $\varepsilon>0, \Gamma$ has an internal matching saturating a set of measure $\geq L(\mu)(X)-\varepsilon$ in $X$ and a set of measure $\geq L(\mu)(X)-\varepsilon$ in $Y$.

At the end we show that the conclusion of Hall's theorem (Proposition 10.1) cannot be improved. We give an example of a $\Sigma_{1}^{0}$ graph (a countable union of internal graphs) which satisfies Hall's condition and which, nevertheless, cannot possess not only an internal matching saturating almost all the domains of the graph but any matching which preserves measure.

Example. Let $T=\left\{1, \ldots, 2^{H}\right\}$ be a hyperfinite set, where $H$ is, as before, an infinite integer. Let $\nu$ be a counting measure on $T$ defined as $\nu(A)=|A| / 2^{H}$ for $A$ internal. We shall define a $\Sigma_{1}^{0}$ graph $\Gamma$

$$
\Gamma \subseteq T \times T,
$$

as follows.

Given the point $2^{H-n} \in T$, where $n$ is a standard integer, we first define the set $L_{n}=\left\{(\alpha, \beta) \in T \times T: \beta=\alpha-2^{H-n}+1\right\}$, which is just the segment in $T \times T$ joining the points $\left(2^{H-n}, 1\right)$ and $\left(2^{H}, 2^{H}-2^{H-n}+1\right)$. Then, we define

$$
\Gamma=\bigcup_{n=1}^{\infty} L_{n} \text {. }
$$

Claim I. $\Gamma$ satisfies Hall's condition.

Proof. Indeed, for every $\varepsilon>0, \Gamma$ possesses an internal matching (namely the segment $L_{n}$ for some suitable $n$ ) of measure defect $\leq \varepsilon$. This implies that $\Gamma$ satisfies Hall's condition.

It is easy to see that $\Gamma$ cannot possess an internal matching of the domain of full measure in $T$. Indeed, if

$$
\mathscr{M} \subseteq \Gamma=\bigcup_{n=1}^{\infty} L_{n},
$$


is an internal matching of $\Gamma$ then, by saturation, $\mathscr{M} \subseteq \bigcup_{k=1}^{n} L_{k}$ for some $n \in \mathbb{N}$, and, therefore, the measure defect of $\mathscr{M}$ must be $\geq 1 / n$.

Moreover, we shall prove that even more general functions, namely those which preserve measure, cannot be matchings of $\Gamma$ saturating almost all of $T$.

Claim II. There does not exist a measure preserving matching $\mathscr{M} \subseteq \Gamma$ in $\Gamma$, i.e.

$$
L(\mu)(\mathscr{M}(A))=L(\mu)(A)
$$

for every internal $A \subseteq T$, such that $L(\mu)(\operatorname{dom}(\mathscr{M}))=L(\mu)(T)$.

Proof. Suppose that we have such a matching $\mathscr{M}$. Let us partition $\mathscr{M}$ into three sets $\mathscr{M}=\mathscr{M}_{0} \cup \mathscr{K} \cup \mathscr{M}_{1}$ by defining

$$
\begin{aligned}
\mathscr{M}_{0} & =\left\{(s, t) \in \mathscr{M}: 1 \leq s \leq 2^{H-1}\right\}, \\
\mathscr{K} & =\left\{(s, t) \in \mathscr{M}: 2^{H-1}<s \leq 2^{H}, 1 \leq t \leq 2^{H-1}\right\}, \\
\mathscr{M}_{1} & =\left\{(s, t) \in \mathscr{M}: 2^{H-1}<s \leq 2^{H}, 2^{H-1}<t \leq 2^{H}\right\} .
\end{aligned}
$$

The set $\mathscr{M}_{0}$ is the part of the matching $\mathscr{M}$ in the triangle $T_{0}$ with vertices $(1,1),\left(2^{H-1}, 1\right)$, and $\left(2^{H-1}, 2^{H-1}\right)$; the set $\mathscr{M}_{1}$ is the part of $\mathscr{M}$ in the triangle $T_{1}$ with vertices $\left(2^{H-1}, 2^{H-1}\right),\left(2^{H}, 2^{H-1}\right)$, and $\left(2^{H}, 2^{H}\right)$; and the set $\mathscr{K}$ is the part of $\mathscr{M}$ in the square $\left\{2^{H-1}, \ldots, 2^{H}\right\} \times\left\{2^{H-1}, \ldots, 2^{H}\right\}$. Let us first note that $\mathscr{M}$ maps Loeb measurable sets into Loeb measurable sets, and that for every measurable set $M, L(\mu)(\mathscr{M}(M))=L(\mu)(M)$. In particular, $L(\mu)(\mathscr{M}(T))=1$.

The fact that the domain of $\mathscr{M}$ is measurable implies that $\operatorname{dom}\left(\mathscr{M}_{1}\right)$ is also measurable. We claim that

$$
L(\mu)\left(\operatorname{dom}\left(\mathscr{M}_{1}\right)\right)=1 / 2 .
$$

Indeed, if $L(\mu)\left(\operatorname{dom}\left(\mathscr{M}_{1}\right)\right)<1 / 2$, then we would also have $L(\mu)\left(\operatorname{ran}\left(\mathscr{M}_{1}\right)\right)<$ $1 / 2$ which contradicts $L(\mu)(T)=1$.

Therefore, $L(\mu)\left(\operatorname{dom}\left(\mathscr{M}_{1}\right)\right)=1 / 2$, and we conclude that

$$
L(\mu)(\operatorname{dom}(\mathscr{K}))=0,
$$

because $L(\mu)\left(\operatorname{dom}\left(\mathscr{M}_{1}\right) \cup \operatorname{dom}(\mathscr{K})\right)=1 / 2$.

We see that the domain of the part $\mathscr{K}$ of our matching $\mathscr{M}$ lying in the square $\left\{2^{H-1}, \ldots, 2^{H}\right\} \times\left\{2^{H-1}, \ldots, 2^{H}\right\}$ is of measure zero, so that we may concentrate on the parts $\mathscr{M}_{0}$ and $\mathscr{M}_{1}$ in the triangles $T_{0}$ and $T_{1}$. But, the part of the graph $\Gamma$ inside the triangle $T_{0}$ and $T_{1}$ respectively, looks exactly alike the original graph $\Gamma$, so we can proceed our operation on $\mathscr{M}_{0}$ and $\mathscr{M}_{1}$ in the same manner as we did on the original matching $\mathscr{M}$. We shall obtain sets $\mathscr{M}_{00}, \mathscr{M}_{01}, \mathscr{M}_{10}, \mathscr{M}_{11}$, and $\mathscr{K}_{0}, \mathscr{K}_{1}$. Generally, for every finite sequence $\mathfrak{s}$ of 0's and 1's we shall obtain a sequence of sets $\mathscr{M}_{\mathfrak{s}}$ and $\mathscr{K}_{\mathfrak{s}}$ and triangles $T_{\mathfrak{s}}$ such that:

(i) the triangles $T_{\mathfrak{s} \wedge}$ and $T_{\mathfrak{s} \wedge}$ are obtained from the triangle $T_{\mathfrak{s}}$ in the same as the triangles $T_{0}$ and $T_{1}$ are obtained from the original triangle $T$;

(ii) $\mathscr{M}_{\mathfrak{s}}$ is the part of the matching $\mathscr{M}$ belonging to the triangle $T_{\mathfrak{s}}$, and $\mathscr{K}_{\mathfrak{s}}$ is the part of the matching $\mathscr{M}$ belonging to the square $K_{\mathfrak{s}}=T_{\mathfrak{s}} \backslash\left(T_{\mathfrak{s} \wedge} \cup T_{\mathfrak{s} \wedge 1}\right)$;

(iii)

$$
L(\mu)\left(\operatorname{dom}\left(\mathscr{K}_{\mathrm{s}}\right)\right)=0 .
$$

Now, it is clear that the graph $\Gamma$ is the union of the parts of $\Gamma$ belonging to the squares $K_{\mathfrak{s}}\left(\mathfrak{s} \in 2^{\omega}\right)$, and, consequently 


$$
\mathscr{M}=\bigcup_{s \in 2^{\omega}} \mathscr{K}_{\mathfrak{s}}
$$

It follows that

$$
\operatorname{dom}(\mathscr{M})=\operatorname{dom}\left(\bigcup_{\mathfrak{s} \in 2^{\omega}} \mathscr{K}_{\mathfrak{s}}\right)=\bigcup_{\mathfrak{s} \in 2^{\omega}} \operatorname{dom}\left(\mathscr{K}_{\mathfrak{s}}\right)
$$

so, by (iii),

$$
L(\mu)(\operatorname{dom}(\mathscr{M})) \leq \sum_{\mathfrak{s} \in 2^{\omega}} L(\mu)\left(\operatorname{dom}\left(\mathscr{K}_{\mathfrak{s}}\right)\right)=0 .
$$

This contradicts the fact that $L(\mu)(\mathscr{M}(T))=1$ and that $\mathscr{M}$ is measure preserving.

We know that Souslin injective functions are measure preserving (for they are a.e. equal to internal injective functions [HeRo]) which gives us the following

Corollary. $\Gamma$ does not possess Souslin matchings saturating almost all $T$.

Open questions. In [ $\left[\mathrm{Zi}_{3}\right]$ the author gives some examples of graphs for which Proposition 10.1 or Proposition 1.2 still remains true. However, the question if the conclusion of Proposition 10.1, for example, still holds for arbitrary Borel or Souslin (or some other) graph satisfying Hall's condition remains open.

\section{ACKNOWLEDGMENTS}

The author is grateful to the referee for many valuable suggestions and remarks.

\section{REFERENCES}

[Bo] B. Bollobas, Extremal graph theory, Academic Press, New York, 1978.

[BoVa] B. Bollobas and N. Th. Varopoulos, Representation of systems of measurable sets, Math. Proc. Cambridge Philos. Soc. 78 (1974), 323-325.

[Ha] P. Hall, On representatives of subsets, J. London Math. Soc. 10 (1935), 26-30.

[He] C. W. Henson, Analytic sets, Baire sets, and the standard part map, Canad. J. Math. 31 (1979), 663-672.

[HeRo] C. W. Henson and D. Ross, Analytic mappings on hyperfinite sets (to appear).

[HPS] M. Holz, K. P. Podewski, and K. Steffens, Injective choice function, Lecture Notes in Math., vol. 1238, Springer-Verlag, 1987.

[HuLo] A. E. Hurd and P. A. Loeb, An introduction to nonstandard real analysis, Academic Press, 1985.

[KKLM] H. J. Keisler, K. Kunen, S. Leth, and A. Miller, Descriptive set theory over hyperfinite sets, J. Symbolic Logic 54 (1989), 1167-1180.

[Ki] H. A. Kirstead, An effective version of Hall's theorem, Proc. Amer. Math. Soc. 88 (1983), 124-128.

[LaRo] D. Landers and L. Rogge, Universal Loeb-measurability of sets and of the standard part map with applications, Trans. Amer. Math. Soc. 304 (1983), 229-243.

[Lo] P. A. Loeb, Conversion from nonstandard to standard measure space and applications in probability theory, Trans. Amer. Math. Soc. 211 (1975), 113-122.

[MaRo] A. B. Manaster and J. G. Rosenstein, Effective matchmaking (recursive theoretic aspects of a theorem of Philip Hall), Proc. London Math. Soc. (3) 25 (1972), 615-654. 
[Mi] L. Mirski, Transversal theory, Academic Press, 1971.

[NW] C. Nash-Williams, Unexplored and semi-explored territories in graph theory, New Directions in Graph Theory, (Frank Harary, ed.), Academic Press, 1973.

[Ra] R. Rado, A theorem on general measure function, Proc. London Math. Soc. 44 (1938), 61-91.

[Ro] D. Ross, (private communication).

[StBa] K. D. Stroyan and J. M. Bayod, Foundations of infinitesimal stochastic analysis, NorthHolland, 1986.

[StLu] K. D. Stroyan and W. A. J. Luxemburg, Introduction to the theory of infinitesimals, Academic Press, 1976.

[Ž $\mathrm{i}_{1}$ ] B. Živaljević, Rado's theorem for the Loeb space of an internal *-finitely additive measure space, Proc. Amer. Math. Soc. 112 (1991), 203-207.

[Ž $\left.\mathrm{i}_{2}\right] \quad$, The structure of graphs all of whose $Y$-sections are internal sets, J. Symbolic Logic 56 (1991), 50-66.

[Ž $\left.\mathrm{i}_{3}\right]$ _ Hyperfinite transversal theory. II (in preparation).

Department of Mathematics, University of Illinois at Urbana-Champaign, Urbana, ILLINOIS 61801

Current address: Department of Computer Science, Michigan State University, East Lansing, Michigan 48824 